Supporting Information

\title{
Phosphine-Catalyzed $\alpha$-Umpolung-Aldol Reaction for the Synthesis of Benzo[b]azapin-3-ones
}

\author{
Kui Zhang, Lingchao Cai, Sooji Hong, and Ohyun Kwon* \\ Department of Chemistry and Biochemistry, University of California, Los Angeles, \\ California 90095-1569, United State
}

Table of Contents

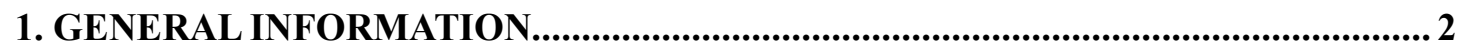

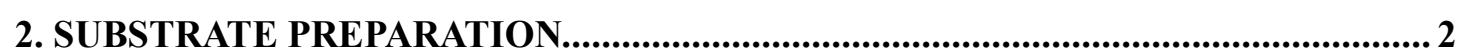

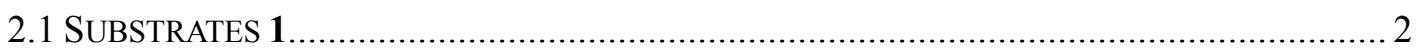

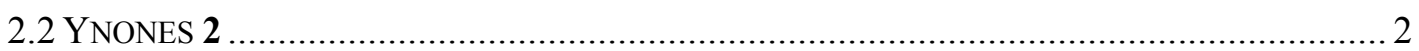

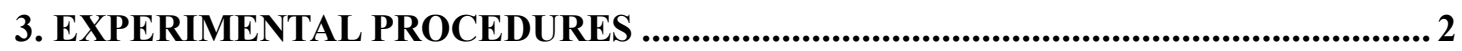

General Procedure for the Synthesis of the Benzo[b]azapin-3-one 3a-s....................... 2

Characterization Data for the Benzo[b]azapin-3-ones 3a-3s..................................... 3

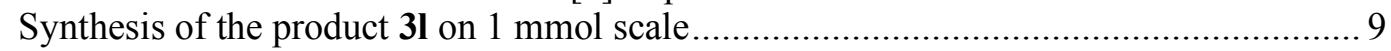

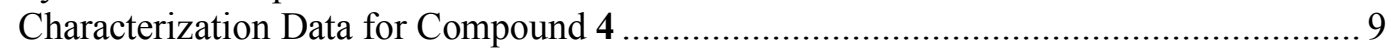

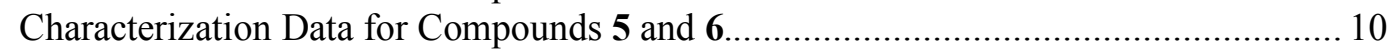

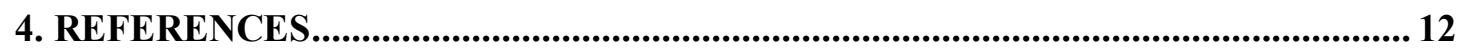

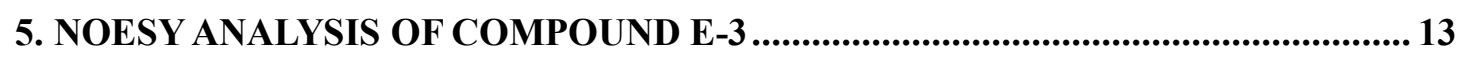

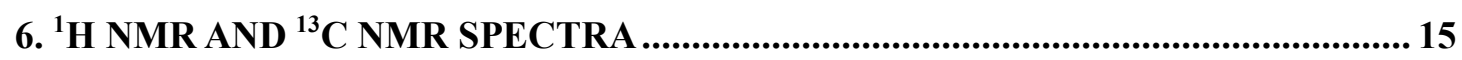

COSY AND NOESY ANALYSIS OF COMPOUND 5. ................................................ 


\section{General Information}

All reactions were performed in dry solvents under an $\mathrm{Ar}$ atmosphere and anhydrous conditions. $\mathrm{CH}_{2} \mathrm{Cl}_{2}$, THF, and $\mathrm{MeCN}$ were freshly distilled over $\mathrm{CaH}_{2}$ prior to use. All other reagents were used as received from commercial sources. Reactions were monitored through thin layer chromatography (TLC) on $0.25-\mathrm{mm}$ SiliCycle silica gel plates and visualized under UV light. Flash column chromatography (FCC) was performed using SiliCycle Silica-P Flash silica gel $(60-\AA \AA$ pore size, $40-63 \mu \mathrm{m})$. IR spectra were recorded using a Jasco FT-IR 4100 spectrometer. NMR spectra were recorded using a Bruker Avance-500 instrument, calibrated to $\mathrm{CD}(\mathrm{H}) \mathrm{Cl}_{3}$ as the internal reference $\left(7.26\right.$ and $77.0 \mathrm{ppm}$ for ${ }^{1} \mathrm{H}$ and ${ }^{13} \mathrm{C}$ NMR spectra, respectively). ${ }^{1} \mathrm{H}$ NMR spectral data are reported in terms of chemical shift $(\delta, \mathrm{ppm})$, multiplicity, coupling constant $(\mathrm{Hz})$, and integration. ${ }^{13} \mathrm{C}$ NMR spectral data are reported in terms of chemical shift $(\delta, \mathrm{ppm})$. The following abbreviations indicate the multiplicities: $\mathrm{s}$, singlet; d, doublet; t, triplet; q, quartet; m, multiplet. High-resolution mass spectra were recorded using a Waters LCT Premier XE time-of-flight instrument controlled by MassLynx 4.1 software. Samples were infused through direct loop injection from a Waters Acquity UPLC into the multi-mode ionization source. The lock mass standard for accurate mass determination was leucine enkephalin (Sigma L9133).

\section{Substrate Preparation}

\subsection{Substrates 1}

The $o$-( $N$-tosyl)benzaldehyde 1a was prepared from the corresponding $o$-aminobenzyl alcohol in two steps, without purification of any intermediates. ${ }^{1}$ The other $o$ - $(N$-tosyl)benzaldehydes were prepared from the corresponding acids in three steps, without purification of any

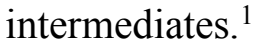

\subsection{Ynones 2}

The ynones 2 were prepared using reported methods. ${ }^{2}$

\section{Experimental Procedures}

\section{General Procedure for the Synthesis of the Benzo[b]azapin-3-one 3a-s}

An $o$-( $N$-tosyl)benzaldehyde $1(0.2 \mathrm{mmol})$, an ynone 2 (0.3 mmol, 1.5 equiv), $\mathrm{Ph}_{2} \mathrm{EtP}(0.04$ mmol, 0.2 equiv), and $n$-propanol $(2 \mathrm{~mL})$ were added sequentially to a flame-dried flask ( 25 $\mathrm{mL}$ ) and then the reaction mixture was stirred at room temperature until the starting material 1 had been consumed. The solvent was evaporated under reduced pressure and the product was purified through silica gel flash column chromatography to yield the desired benzo[b]azapin-3-one. 


\section{Characterization Data for the Benzo[b]azapin-3-ones 3a-3s}

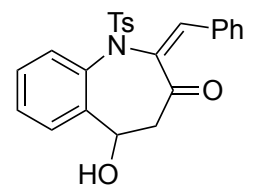

Following the general procedure, 3a $(62.5 \mathrm{mg}, 74 \%)$ was obtained as a pale solid from $\mathrm{N}$-(2-formylphenyl)-4-methylbenzenesulfonamide 1a (55.0 mg, $0.2 \mathrm{mmol})$; m.p. $178-180{ }^{\circ} \mathrm{C}$; $R_{\mathrm{f}}=0.36$ (hexane/EtOAc, 3:1); FCC (hexanes/ethyl acetate 10:1 to 3:1); IR (ATP) $v_{\max } 3250$, 2927, 1727, 1335, 1247, 1178, $1092 \mathrm{~cm}^{-1} ;{ }^{1} \mathrm{H}$ NMR (500 MHz, $\left.\mathrm{CDCl}_{3}\right) \delta 7.75-7.73(\mathrm{~m}, 2 \mathrm{H})$, 7.61-7.59 (m, 2H), 7.38-7.35 (m, 3H), 7.31-7.22 (m, 5H), 7.03-7.01 (m, 1H), $6.74(\mathrm{~s}, 1 \mathrm{H})$, $6.42(\mathrm{~s}, 1 \mathrm{H}), 5.96(\mathrm{t}, J=7.9 \mathrm{~Hz}, 1 \mathrm{H}), 3.12(\mathrm{dd}, J=7.9,18.5 \mathrm{~Hz}, 1 \mathrm{H}), 2.62(\mathrm{dd}, J=7.9,18.5$ $\mathrm{Hz}, 1 \mathrm{H}), 2.40(\mathrm{~s}, 3 \mathrm{H}) ;{ }^{13} \mathrm{C}$ NMR $\left(125 \mathrm{MHz}, \mathrm{CDCl}_{3}\right) \delta 198.4,146.7,144.3,136.1$ (2C), 133.6, 133.3, 130.3, 129.8, 129.3, 128.7, 128.6, 127.7, 127.4, 126.8, 126.3, 106.0, 76.4, 41.8, 21.6; HRMS (ESI-TOF) calcd for $\mathrm{C}_{24} \mathrm{H}_{22} \mathrm{NO}_{4} \mathrm{~S}[\mathrm{M}+\mathrm{H}]^{+} \mathrm{m} / z$ 420.1270, found 420.1283.

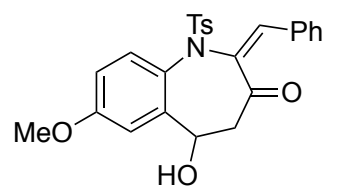

Following the general procedure, $3 \mathbf{b}(73.6 \mathrm{mg}, 82 \%)$ was obtained as a pale solid from $\mathrm{N}$-(2-formyl-4-methoxyphenyl)-4-methylbenzenesulfonamide $\mathbf{1 b}(61.0 \mathrm{mg}, 0.2 \mathrm{mmol}) ; \mathrm{m} . \mathrm{p}$. $154-156{ }^{\circ} \mathrm{C} ; R_{\mathrm{f}}=0.32$ (hexane/EtOAc, 3:1); FCC (hexanes/ethyl acetate 10:1 to 3:1); IR (ATP) $v_{\max } 3258,2932,1730,1321,1499,1326,1294,1244,1180,1092 \mathrm{~cm}^{-1} ;{ }^{1} \mathrm{H}$ NMR (500 $\left.\mathrm{MHz}, \mathrm{CDCl}_{3}\right) \delta 7.76-7.74(\mathrm{~m}, 2 \mathrm{H}), 7.60-7.58(\mathrm{~m}, 2 \mathrm{H}), 7.37-7.34(\mathrm{~m}, 2 \mathrm{H}), 7.29-7.25(\mathrm{~m}, 3 \mathrm{H})$, $6.94(\mathrm{~s}, 1 \mathrm{H}), 6.72-6.67(\mathrm{~m}, 2 \mathrm{H}), 6.47(\mathrm{~s}, 1 \mathrm{H}), 6.39(\mathrm{~s}, 1 \mathrm{H}), 6.06(\mathrm{t}, J=7.9 \mathrm{~Hz}, 1 \mathrm{H}), 3.71(\mathrm{~s}$, $3 \mathrm{H}), 3.19$ (dd, $J=7.9,18.5 \mathrm{~Hz}, 1 \mathrm{H}), 2.57(\mathrm{dd}, J=7.9,18.5 \mathrm{~Hz}, 1 \mathrm{H}), 2.40(\mathrm{~s}, 3 \mathrm{H}) ;{ }^{13} \mathrm{C} \mathrm{NMR}$ $\left(125 \mathrm{MHz}, \mathrm{CDCl}_{3}\right) \delta 198.8,159.5,147.0,144.2,140.7,136.0,133.5,130.3,129.8,129.6$, 128.7, 128.4, 127.5, 125.2, 114.1, 111.8, 105.5, 75.8, 55.5, 42.4, 21.6; HRMS (ESI-TOF) calcd for $\mathrm{C}_{25} \mathrm{H}_{24} \mathrm{NO}_{5} \mathrm{~S}[\mathrm{M}+\mathrm{H}]^{+} m / z 450.1375$, found 450.1368 .

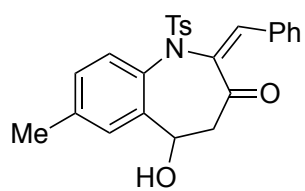

Following the general procedure, 3c $(66.7 \mathrm{mg}, 77 \%)$ was obtained as a pale solid from $N$-(2-formyl-4-methylphenyl)-4-methylbenzenesulfonamide 1c $(57.8 \mathrm{mg}, 0.2 \mathrm{mmol})$; m.p. $144{ }^{\circ} \mathrm{C} ; R_{\mathrm{f}}=0.38$ (hexane/EtOAc, 3:1); FCC (hexanes/ethyl acetate 10:1 to 3:1); IR (ATP) $\nu_{\max } 3256,2936,1734,1345,1283,1223,1145,1077 \mathrm{~cm}^{-1} ;{ }^{1} \mathrm{H}$ NMR $\left(500 \mathrm{MHz}, \mathrm{CDCl}_{3}\right) \delta$ 7.75-7.73 (m, 2H), 7.60-7.58 (m, 2H), 7.38-7.35 (m, 2H), 7.30-7.25 (m, 3H), $7.17(\mathrm{~s}, 1 \mathrm{H})$, $7.04-7.01(\mathrm{~m}, 1 \mathrm{H}), 6.83-6.81(\mathrm{~m}, 1 \mathrm{H}), 6.52(\mathrm{~s}, 1 \mathrm{H}), 6.41(\mathrm{~s}, 1 \mathrm{H}), 5.96(\mathrm{t}, J=7.9 \mathrm{~Hz}, 1 \mathrm{H})$, $3.11(\mathrm{dd}, J=7.9,18.5 \mathrm{~Hz}, 1 \mathrm{H}), 2.59(\mathrm{dd}, J=7.9,18.5 \mathrm{~Hz}, 1 \mathrm{H}), 2.40(\mathrm{~s}, 3 \mathrm{H}), 2.40(\mathrm{~s}, 3 \mathrm{H}) ;{ }^{13} \mathrm{C}$ NMR $\left(125 \mathrm{MHz}, \mathrm{CDCl}_{3}\right) \delta 198.6,146.8,144.2,138.2,136.7,136.2,133.4,130.7,130.3$, $130.0,129.8,128.7,128.5,127.4,127.3,126.9,105.7,76.2,42.0,21.6,21.2$; HRMS (ESI-TOF) calcd for $\mathrm{C}_{25} \mathrm{H}_{24} \mathrm{NO}_{4} \mathrm{~S}[\mathrm{M}+\mathrm{H}]^{+} m / z$ 434.1426, found 434.1430. 


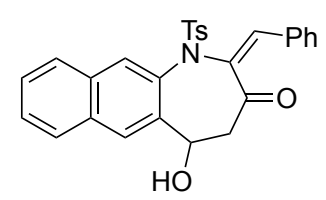

Following the general procedure, $3 \mathbf{d}(65.7 \mathrm{mg}, 70 \%)$ was obtained as a pale solid from $N$-(3-formylnaphthalen-2-yl)-4-methylbenzenesulfonamide 1d (65.0 mg, $0.2 \mathrm{mmol})$; m.p. $140-142{ }^{\circ} \mathrm{C} ; R_{\mathrm{f}}=0.40$ (hexane/EtOAc, 3:1); FCC (hexanes/ethyl acetate 10:1 to 3:1); IR (ATP) $v_{\max } 3263,2930,1735,1327,1279,1153,1087 \mathrm{~cm}^{-1} ;{ }^{1} \mathrm{H}$ NMR $\left(500 \mathrm{MHz}, \mathrm{CDCl}_{3}\right) \delta$ $7.81(\mathrm{~s}, 1 \mathrm{H}), 7.78-7.75(\mathrm{~m}, 3 \mathrm{H}), 7.69-7.64(\mathrm{~m}, 3 \mathrm{H}), 7.59(\mathrm{~s}, 1 \mathrm{H}), 7.50-7.45(\mathrm{~m}, 2 \mathrm{H})$, $7.41-7.38(\mathrm{~m}, 2 \mathrm{H}), 7.33-7.30(\mathrm{~m}, 1 \mathrm{H}), 7.24-7.22(\mathrm{~m}, 2 \mathrm{H}), 6.47(\mathrm{~s}, 1 \mathrm{H}), 6.00(\mathrm{t}, J=7.9 \mathrm{~Hz}$, $1 \mathrm{H}), 3.16(\mathrm{dd}, J=7.9,18.5 \mathrm{~Hz}, 1 \mathrm{H}), 2.74(\mathrm{dd}, J=7.9,18.5 \mathrm{~Hz}, 1 \mathrm{H}), 2.39(\mathrm{~s}, 3 \mathrm{H}) ;{ }^{13} \mathrm{C} \mathrm{NMR}$ $\left(125 \mathrm{MHz}, \mathrm{CDCl}_{3}\right) \delta 198.1,146.4,144.3,136.3,133.3,133.2,133.1,131.6,131.5,130.3$, $129.9,128.8,128.7,128.0,127.5,127.4,127.3,126.8,126.4,124.2,106.4,77.1,41.4,21.6$; HRMS (ESI-TOF) calcd for $\mathrm{C}_{28} \mathrm{H}_{24} \mathrm{NO}_{4} \mathrm{~S}[\mathrm{M}+\mathrm{H}]^{+} \mathrm{m} / z$ 470.1426, found 470.1444 .

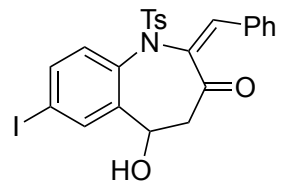

Following the general procedure, $3 \mathbf{e}(81.6 \mathrm{mg}, 75 \%)$ was obtained as a pale solid from $\mathrm{N}$-(2-formyl-4-iodophenyl)-4-methylbenzenesulfonamide 1e $(80.2 \mathrm{mg}, 0.2 \mathrm{mmol}) ; \mathrm{m} . \mathrm{p}$. 154-156 ${ }^{\circ} \mathrm{C} ; R_{\mathrm{f}}=0.41$ (hexane/EtOAc, 3:1); FCC (hexanes/ethyl acetate 10:1 to 3:1); IR (ATP) $v_{\max } 3259,2928,1730,1335,1246,1178,1162,1092 \mathrm{~cm}^{-1} ;{ }^{1} \mathrm{H}$ NMR $(500 \mathrm{MHz}$, $\left.\mathrm{CDCl}_{3}\right) \delta 7.73-7.72(\mathrm{~m}, 2 \mathrm{H}), 7.67(\mathrm{~s}, 1 \mathrm{H}), 7.61-7.55(\mathrm{~m}, 3 \mathrm{H}), 7.40-7.37(\mathrm{~m}, 2 \mathrm{H}), 7.33-7.30$ $(\mathrm{m}, 1 \mathrm{H}), 7.27(\mathrm{~s}, 1 \mathrm{H}), 6.82-6.80(\mathrm{~m}, 1 \mathrm{H}), 6.44(\mathrm{~s}, 1 \mathrm{H}), 5.82(\mathrm{t}, J=7.9 \mathrm{~Hz}, 1 \mathrm{H}), 3.09(\mathrm{dd}, J=$ 7.9, $18.5 \mathrm{~Hz}, 1 \mathrm{H}), 2.54(\mathrm{dd}, J=7.9,18.5 \mathrm{~Hz}, 1 \mathrm{H}), 2.40(\mathrm{~s}, 3 \mathrm{H}) ;{ }^{13} \mathrm{C} \mathrm{NMR}\left(125 \mathrm{MHz}, \mathrm{CDCl}_{3}\right)$ $\delta 197.5,146.2,144.6,138.5,137.3,136.0,135.9,133.7,133.1,130.3,130.0,128.8,127.6$, 127.3, 106.7, 92.1, 76.0, 41.5, 21.6; HRMS (ESI-TOF) calcd for $\mathrm{C}_{24} \mathrm{H}_{21} \mathrm{INO}_{4} \mathrm{~S}[\mathrm{M}+\mathrm{H}]^{+} \mathrm{m} / \mathrm{z}$ 546.0236 , found 546.0245 .

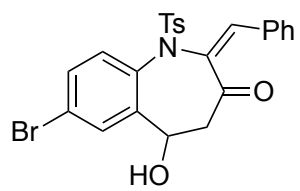

Following the general procedure, $3 f(72.6 \mathrm{mg}, 73 \%)$ was obtained as a pale solid from $\mathrm{N}$-(4-bromo-2-formylphenyl)-4-methylbenzenesulfonamide 1 ff (70.6 mg, $0.2 \mathrm{mmol}$ ); m.p. $154{ }^{\circ} \mathrm{C} ; R_{\mathrm{f}}=0.41$ (hexane/EtOAc, 3:1); FCC (hexanes/ethyl acetate $10: 1$ to $3: 1$ ); IR (ATP) $\nu_{\max } 3263,2936,1729,1335,1248,1163,1090 \mathrm{~cm}^{-1} ;{ }^{1} \mathrm{H}$ NMR $\left(500 \mathrm{MHz}, \mathrm{CDCl}_{3}\right) \delta 7.73(\mathrm{~d}, J$ $=7.3 \mathrm{~Hz}, 2 \mathrm{H}), 7.59(\mathrm{~d}, J=8.3 \mathrm{~Hz}, 2 \mathrm{H}), 7.50(\mathrm{~d}, J=2.3 \mathrm{~Hz}, 1 \mathrm{H}), 7.40-7.36(\mathrm{~m}, 3 \mathrm{H})$, $7.33-7.30(\mathrm{~m}, 1 \mathrm{H}), 7.27$ (d, $J=8.1 \mathrm{~Hz}, 2 \mathrm{H}), 6.91(\mathrm{~d}, J=8.6 \mathrm{~Hz}, 1 \mathrm{H}), 6.63(\mathrm{~s}, 1 \mathrm{H}), 6.45$ (s, $1 \mathrm{H}), 5.86(\mathrm{t}, J=8.0 \mathrm{~Hz}, 1 \mathrm{H}), 3.11(\mathrm{dd}, J=7.8,18.5 \mathrm{~Hz}, 1 \mathrm{H}), 2.55(\mathrm{dd}, J=8.3,18.5 \mathrm{~Hz}, 1 \mathrm{H})$, $2.41(\mathrm{~s}, 3 \mathrm{H}) ;{ }^{13} \mathrm{C}$ NMR $\left[125 \mathrm{MHz},\left(\mathrm{CD}_{3}\right)_{2} \mathrm{CO}\right] \delta 197.5,146.2,144.6,137.8,135.9,133.1$, 132.8, 132.5, 130.3, 130.1, 130.0, 128.8 (2C), 127.8, 127.3, 121.3, 106.7, 76.0, 41.6, 21.6; HRMS (ESI-TOF) calcd for $\mathrm{C}_{24} \mathrm{H}_{21} \mathrm{BrNO}_{4} \mathrm{~S}[\mathrm{M}+\mathrm{H}]^{+} \mathrm{m} / z$ 498.0375, found 498.0385. 


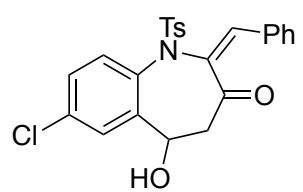

Following the general procedure, $3 \mathrm{~g}(63.4 \mathrm{mg}, 70 \%)$ was obtained as a pale solid from $N$-(4-chloro-2-formylphenyl)-4-methylbenzenesulfonamide $1 \mathrm{~g}(61.8 \mathrm{mg}, 0.2 \mathrm{mmol}) ; \mathrm{m} . \mathrm{p}$. $158{ }^{\circ} \mathrm{C} ; R_{\mathrm{f}}=0.35$ (hexane/EtOAc, 3:1); FCC (hexanes/ethyl acetate 10:1 to 3:1); IR (ATP) $v_{\max } 3252,2927,1710,1321,1282,1157,1044 \mathrm{~cm}^{-1} ;{ }^{1} \mathrm{H}$ NMR $\left(500 \mathrm{MHz}, \mathrm{CDCl}_{3}\right) \delta 7.73(\mathrm{~d}, J$ $=7.4 \mathrm{~Hz}, 2 \mathrm{H}), 7.59(\mathrm{~d}, J=8.3 \mathrm{~Hz}, 2 \mathrm{H}), 7.40-7.36(\mathrm{~m}, 3 \mathrm{H}), 7.33-7.27(\mathrm{~m}, 3 \mathrm{H}), 7.22-7.20(\mathrm{~m}$, $1 \mathrm{H}), 6.95(\mathrm{~d}, J=8.6 \mathrm{~Hz}, 1 \mathrm{H}), 6.61(\mathrm{~s}, 1 \mathrm{H}), 6.44(\mathrm{~s}, 1 \mathrm{H}), 5.89(\mathrm{t}, J=8.0 \mathrm{~Hz}, 1 \mathrm{H}), 3.12(\mathrm{dd}, J=$ 7.9, $18.4 \mathrm{~Hz}, 1 \mathrm{H}), 2.55(\mathrm{dd}, J=8.2,18.5 \mathrm{~Hz}, 1 \mathrm{H}), 2.41(\mathrm{~s}, 3 \mathrm{H}) ;{ }^{13} \mathrm{C} \mathrm{NMR}\left(125 \mathrm{MHz}, \mathrm{CDCl}_{3}\right)$ $\delta$ 197.6, 146.2, 144.6, 138.0, 135.9, 133.6, 133.1, 132.1, 130.3, 129.4, 128.8, 127.9, 127.4, 127.1, 106.6, 76.0, 41.6, 21.6; HRMS (ESI-TOF) calcd for $\mathrm{C}_{24} \mathrm{H}_{21} \mathrm{ClNO}_{4} \mathrm{~S}[\mathrm{M}+\mathrm{H}]^{+} \mathrm{m} / \mathrm{z}$ 454.0880 , found 454.0889 .

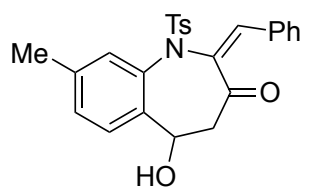

Following the general procedure, $\mathbf{3 h}(62.4 \mathrm{mg}, 72 \%)$ was obtained as a colorless oil from $N$-(2-formyl-5-methylphenyl)-4-methylbenzenesulfonamide $1 \mathrm{~h}(57.8 \mathrm{mg}, 0.2 \mathrm{mmol}) ; R_{\mathrm{f}}=$ 0.40 (hexane/EtOAc, 3:1); FCC (hexanes/ethyl acetate 10:1 to 3:1); IR (ATP) $v_{\max } 3262$, 2938, $1735,1346,1283,1230,1146,1078 \mathrm{~cm}^{-1} ;{ }^{1} \mathrm{H}$ NMR $\left(500 \mathrm{MHz}, \mathrm{CDCl}_{3}\right) \delta 7.73-7.71(\mathrm{~m}, 2 \mathrm{H})$, 7.61-7.60 (m, 2H), 7.38-7.35 (m, 2H), 7.30-7.26 (m, 2H), 7.24-7.21 (m, 2H), 7.07 (d, $J=7.8$ $\mathrm{Hz}, 1 \mathrm{H}), 6.89$ (s, 1H), $6.40(\mathrm{~s}, 1 \mathrm{H}), 5.83(\mathrm{t}, J=7.9 \mathrm{~Hz}, 1 \mathrm{H}), 3.03(\mathrm{dd}, J=7.9,18.5 \mathrm{~Hz}, 1 \mathrm{H})$, $2.59(\mathrm{dd}, J=7.9,18.5 \mathrm{~Hz}, 1 \mathrm{H}), 2.40(\mathrm{~s}, 3 \mathrm{H}), 2.25(\mathrm{~s}, 3 \mathrm{H}) ;{ }^{13} \mathrm{C}$ NMR $\left(125 \mathrm{MHz}, \mathrm{CDCl}_{3}\right) \delta$ $198.4,146.6,144.2,139.7,136.3,133.7,133.3,132.3,130.2,129.8,128.7,128.5,128.2$, 127.3, 126.8, 126.7, 105.9, 76.4, 41.6, 21.6, 21.1; HRMS (ESI-TOF) calcd for $\mathrm{C}_{25} \mathrm{H}_{24} \mathrm{NO}_{4} \mathrm{~S}$ $[\mathrm{M}+\mathrm{H}]^{+} \mathrm{m} / \mathrm{z}$ 434.1426, found 434.1434.

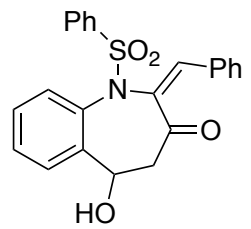

Following the general procedure, $3 \mathbf{i}(51.0 \mathrm{mg}, 63 \%)$ was obtained as a pale solid from $N$-(2-formylphenyl)benzenesulfonamide $1 \mathbf{i}(52.2 \mathrm{mg}, 0.2 \mathrm{mmol}) ; \mathrm{m} . \mathrm{p} .146{ }^{\circ} \mathrm{C} ; R_{\mathrm{f}}=0.40$ (hexane/EtOAc, 3:1); FCC (hexanes/ethyl acetate 10:1 to 3:1); IR (ATP) $v_{\max } 3259$, 2936, 1728, 1332, 1248, 1179, 1163, $1092 \mathrm{~cm}^{-1} ;{ }^{1} \mathrm{H}$ NMR (500 MHz, $\left.\mathrm{CDCl}_{3}\right) \delta 7.74-7.72(\mathrm{~m}, 4 \mathrm{H})$, 7.60-7.57 (m, 1H), 7.48-7.45 (m, 2H), 7.38-7.35 (m, 3H), 7.31-7.27 (m, 2H), 7.25-7.21 (m, $1 \mathrm{H}), 7.02-7.00(\mathrm{~m}, 1 \mathrm{H}), 6.42(\mathrm{~s}, 1 \mathrm{H}), 5.95$ (t, $J=7.9 \mathrm{~Hz}, 1 \mathrm{H}), 3.11(\mathrm{dd}, J=7.9,18.5 \mathrm{~Hz}, 1 \mathrm{H})$, $2.59(\mathrm{dd}, J=7.9,18.5 \mathrm{~Hz}, 1 \mathrm{H}) ;{ }^{13} \mathrm{C} \mathrm{NMR}\left(125 \mathrm{MHz}, \mathrm{CDCl}_{3}\right) \delta 198.3,146.6,139.1,136.0$, 133.6, 133.3 (2C), 130.3, 129.4, 129.3, 128.7, 128.6, 127.8, 127.3, 126.9, 126.4, 106.0, 76.4, 41.8; HRMS (ESI-TOF) calcd for $\mathrm{C}_{23} \mathrm{H}_{20} \mathrm{NO}_{4} \mathrm{~S}[\mathrm{M}+\mathrm{H}]^{+} \mathrm{m} / z$ 406.1113, found 406.1123 . 


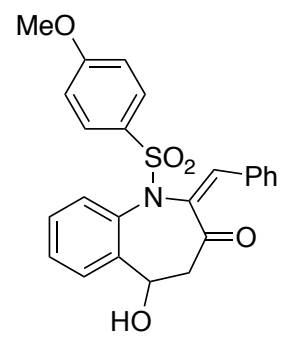

Following the general procedure, $\mathbf{3 j}(56.6 \mathrm{mg}, 65 \%)$ was obtained as a pale solid from $N$-(2-formylphenyl)-4-methoxybenzenesulfonamide $\mathbf{1 j} \quad(58.2 \mathrm{mg}, \quad 0.2 \mathrm{mmol}) ; \quad \mathrm{m} . \mathrm{p}$. $162-164{ }^{\circ} \mathrm{C} ; R_{\mathrm{f}}=0.50$ (hexane/EtOAc, 3:1); FCC (hexanes/ethyl acetate 10:1 to 3:1); IR (ATP) $v_{\max } 3290,2930,1722,1599,1509,1332,1245,1161,1092 \mathrm{~cm}^{-1} ;{ }^{1} \mathrm{H}$ NMR (500 MHz, $\left.\mathrm{CDCl}_{3}\right) \delta$ 7.75-7.73 (m, 2H), 7.66-7.64 (m, 2H), 7.38-7.35 (m, 3H), 7.31-7.22 (m, 3H), $7.02-7.00(\mathrm{~m}, 1 \mathrm{H}), 6.92-6.90(\mathrm{~m}, 2 \mathrm{H}), 6.74(\mathrm{~s}, 1 \mathrm{H}), 6.42(\mathrm{~s}, 1 \mathrm{H}), 6.00(\mathrm{t}, J=7.9 \mathrm{~Hz}, 1 \mathrm{H})$, $3.84(\mathrm{~s}, 3 \mathrm{H}), 3.16(\mathrm{dd}, J=7.9,18.5 \mathrm{~Hz}, 1 \mathrm{H}), 2.63(\mathrm{dd}, J=7.9,18.5 \mathrm{~Hz}, 1 \mathrm{H}) ;{ }^{13} \mathrm{C}$ NMR $(125$ $\left.\mathrm{MHz}, \mathrm{CDCl}_{3}\right) \delta 198.4,163.4,146.6,136.1,133.7,133.3,130.6,130.3,129.5,129.3,128.7$, 128.6, 127.7, 126.8, 126.3, 114.3, 106.0, 76.4, 55.7, 41.8; HRMS (ESI-TOF) calcd for $\mathrm{C}_{24} \mathrm{H}_{22} \mathrm{NO}_{5} \mathrm{~S}[\mathrm{M}+\mathrm{H}]^{+} m / z$ 436.1219, found 436.1217.

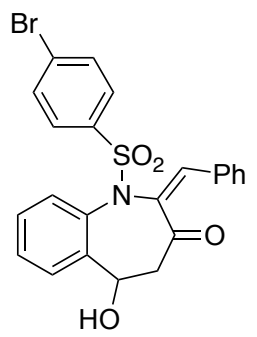

Following the general procedure, 3k $(57 \mathrm{mg}, 59 \%)$ was obtained as a yellow oil from 4-bromo- $N$-(2-formylphenyl)benzenesulfonamide $1 \mathbf{k}(67.8 \mathrm{mg}, 0.2 \mathrm{mmol}) ; \quad R_{\mathrm{f}}=0.47$ (hexane/EtOAc, 3:1); FCC (hexanes/ethyl acetate 10:1 to 3:1); IR (ATP) $v_{\max } 3243,2934$, 1731, 1335, 1248, 1179, 1162, $1092 \mathrm{~cm}^{-1} ;{ }^{1} \mathrm{H}$ NMR (500 MHz, $\left.\mathrm{CDCl}_{3}\right) \delta 7.74(\mathrm{~d}, J=7.6 \mathrm{~Hz}$, 2H), 7.62-7.57 (m, 4H), 7.40-7.36 (m, 3H), 7.32-7.28 (m, 2H), $6.98(\mathrm{~d}, J=7.6 \mathrm{~Hz}, 1 \mathrm{H}), 6.77$ $(\mathrm{s}, 1 \mathrm{H}), 6.44(\mathrm{~s}, 1 \mathrm{H}), 5.99(\mathrm{t}, J=7.9 \mathrm{~Hz}, 1 \mathrm{H}), 3.14(\mathrm{dd}, J=7.9,18.5 \mathrm{~Hz}, 1 \mathrm{H}), 2.69(\mathrm{dd}, J=$ 8.0, $18.5 \mathrm{~Hz}, 1 \mathrm{H}) ;{ }^{13} \mathrm{C} \mathrm{NMR}\left(125 \mathrm{MHz}, \mathrm{CDCl}_{3}\right) \delta 198.0,146.5,138.1,135.9,133.3,133.2$, $132.5,130.3,129.5,128.9,128.8,128.7,128.5,127.9,127.2,126.0,106.2,76.5,41.7$; HRMS (ESI-TOF) calcd for $\mathrm{C}_{23} \mathrm{H}_{19} \mathrm{BrNO}_{4} \mathrm{~S}[\mathrm{M}+\mathrm{H}]^{+} \mathrm{m} / z$ 484.0218, found 484.0222.

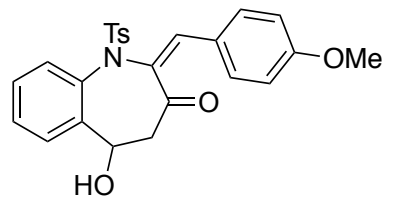

Following the general procedure, 31 (84.4 mg, 94\%) was obtained as a pale solid from $\mathrm{N}$-(2-formylphenyl)-4-methylbenzenesulfonamide 1a (55.0 mg, $0.2 \mathrm{mmol})$; m.p. $134-136{ }^{\circ} \mathrm{C}$; $R_{\mathrm{f}}=0.45$ (hexane/EtOAc, 3:1); FCC (hexanes/ethyl acetate 10:1 to 3:1); IR (ATP) $v_{\max } 3270$, 2930, 1729, 1596, 1496, 1333, 1246, 1157, $1093 \mathrm{~cm}^{-1} ;{ }^{1} \mathrm{H}$ NMR $\left(500 \mathrm{MHz}, \mathrm{CDCl}_{3}\right) \delta$ 7.70-7.68 (m, 2H), 7.61-7.60 (m, 2H), 7.36-7.34 (m, 1H), 7.26-7.23 (m, 3H), 7.09-7.07 (m, $1 \mathrm{H}), 6.92-6.89(\mathrm{~m}, 3 \mathrm{H}), 6.41(\mathrm{~s}, 1 \mathrm{H}), 5.88(\mathrm{t}, J=7.9 \mathrm{~Hz}, 1 \mathrm{H}), 3.82(\mathrm{~s}, 3 \mathrm{H}), 3.07(\mathrm{dd}, J=7.9$, $18.5 \mathrm{~Hz}, 1 \mathrm{H}), 2.57$ (dd, $J=7.9,18.5 \mathrm{~Hz}, 1 \mathrm{H}), 2.39$ (s, 3H); ${ }^{13} \mathrm{C} \mathrm{NMR}\left(125 \mathrm{MHz}, \mathrm{CDCl}_{3}\right) \delta$ 
198.0, 160.0, 145.3, 144.3, 136.2, 135.6, 133.8, 132.0, 129.8, 129.3, 127.4, 127.3, 126.9, 126.0 (2C), 114.3, 106.4, 76.4, 55.3, 41.8, 21.6; HRMS (ESI-TOF) calcd for $\mathrm{C}_{25} \mathrm{H}_{24} \mathrm{NO}_{5} \mathrm{~S}$ [M $+\mathrm{H}]^{+} \mathrm{m} / z$ 450.1375, found 450.1387 .

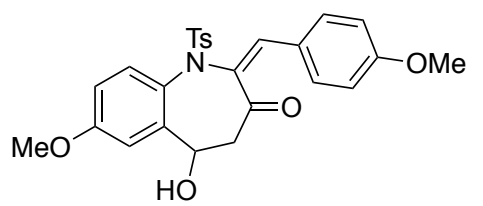

Following the general procedure, $\mathbf{3 m}(94.8 \mathrm{mg}, 99 \%)$ was obtained as a yellow oil from $N$-(2-formyl-4-methoxyphenyl)-4-methylbenzenesulfonamide $1 \mathbf{b}(61.0 \mathrm{mg}, 0.2 \mathrm{mmol}) ; R_{\mathrm{f}}=$ 0.52 (hexane/EtOAc, 3:1); FCC (hexanes/ethyl acetate 10:1 to 3:1); IR (ATP) $v_{\max } 3192,3065$, 1732, 1607, 1494, 1158, $1091 \mathrm{~cm}^{-1} ;{ }^{1} \mathrm{H}$ NMR (500 MHz, $\left.\mathrm{CDCl}_{3}\right) \delta 7.72-7.70(\mathrm{~m}, 2 \mathrm{H})$, 7.59-7.58 (m, 2H), 7.26-7.25 (m, 2H), 6.93-6.92 (m, 1H), 6.90-6.88 (m, 2H), 6.75-6.74 (m, $1 \mathrm{H}), 6.70-6.68(\mathrm{~m}, 1 \mathrm{H}), 6.48(\mathrm{~s}, 1 \mathrm{H}), 6.38(\mathrm{~s}, 1 \mathrm{H}), 5.98(\mathrm{t}, J=7.9 \mathrm{~Hz}, 1 \mathrm{H}), 3.82(\mathrm{~s}, 3 \mathrm{H})$, $3.71(\mathrm{~s}, 3 \mathrm{H}), 3.14(\mathrm{dd}, J=7.9,18.5 \mathrm{~Hz}, 1 \mathrm{H}), 2.53(\mathrm{dd}, J=7.9,18.5 \mathrm{~Hz}, 1 \mathrm{H}), 2.40(\mathrm{~s}, 3 \mathrm{H}) ;{ }^{13} \mathrm{C}$ NMR $\left(125 \mathrm{MHz}, \mathrm{CDCl}_{3}\right) \delta 198.5,159.8,159.4,145.7,144.2,140.5,136.1,131.9,129.8$, $129.5,127.5,126.2,125.3,114.2$, 114.1, 111.7, 105.8, 75.6, 55.5, 55.3, 42.4, 21.6; HRMS (ESI-TOF) calcd for $\mathrm{C}_{26} \mathrm{H}_{26} \mathrm{NO}_{6} \mathrm{~S}[\mathrm{M}+\mathrm{H}]^{+} \mathrm{m} / z$ 480.1481, found 480.1490 .

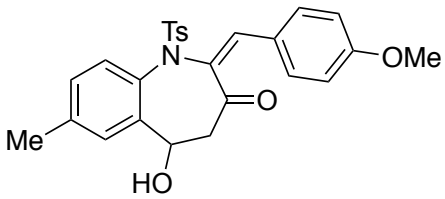

Following the general procedure, $3 \mathbf{n}(88.0 \mathrm{mg}$, 95\%) was obtained as a yellow oil from $\mathrm{N}$-(2-formyl-4-methylphenyl)-4-methylbenzenesulfonamide 1c $(57.8 \mathrm{mg}, 0.2 \mathrm{mmol}) ; R_{\mathrm{f}}=$ 0.28 (hexane/EtOAc, 3:1); FCC (hexanes/ethyl acetate 10:1 to 3:1); IR (ATP) $v_{\max } 3262,3021$, 2971, 1732, 1510, 1275, 1168, $1085 \mathrm{~cm}^{-1} ;{ }^{1} \mathrm{H}$ NMR (500 MHz, $\left.\mathrm{CDCl}_{3}\right) \delta 7.70-7.69(\mathrm{~m}, 2 \mathrm{H})$, 7.60-7.59 (m, 2H), $7.24(\mathrm{~s}, 1 \mathrm{H}), 7.16(\mathrm{~s}, 1 \mathrm{H}), 7.03-7.01(\mathrm{~m}, 1 \mathrm{H}), 6.93-6.87(\mathrm{~m}, 3 \mathrm{H}), 6.73(\mathrm{~s}$, $1 \mathrm{H}), 6.39(\mathrm{~s}, 1 \mathrm{H}), 5.89(\mathrm{t}, J=7.9 \mathrm{~Hz}, 1 \mathrm{H}), 3.82(\mathrm{~s}, 3 \mathrm{H}), 3.06(\mathrm{dd}, J=7.9,18.5 \mathrm{~Hz}, 1 \mathrm{H}), 2.55$ $(\mathrm{dd}, J=7.9,18.5 \mathrm{~Hz}, 1 \mathrm{H}), 2.39(\mathrm{~s}, 3 \mathrm{H}), 2.30(\mathrm{~s}, 3 \mathrm{H}) ;{ }^{13} \mathrm{C} \mathrm{NMR}\left(125 \mathrm{MHz}, \mathrm{CDCl}_{3}\right) \delta 198.4$, $159.9,145.6,144.1,138.0,136.5,136.3,131.9,130.8,129.9,129.8,127.4,127.3,126.8$, 126.1, 114.2, 106.0, 76.1, 55.3, 42.1, 21.6, 21.2; HRMS (ESI-TOF) calcd for $\mathrm{C}_{26} \mathrm{H}_{26} \mathrm{NO}_{5} \mathrm{~S}$ [M $+\mathrm{H}]^{+} \mathrm{m} / z$ 464.1532, found 464.1537 .

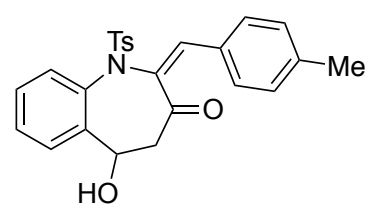

Following the general procedure, $30(78.8 \mathrm{mg}, 91 \%)$ was obtained as a pale solid from $\mathrm{N}$-(2-formylphenyl)-4-methylbenzenesulfonamide 1a (55.0 mg, $0.2 \mathrm{mmol})$; m.p. $154-156{ }^{\circ} \mathrm{C}$; $R_{\mathrm{f}}=0.42$ (hexane/EtOAc, 3:1); FCC (hexanes/ethyl acetate 10:1 to 3:1); IR (ATP) $v_{\max } 3263$, 2928, 1728, 1634, 1333, 1251, 1160, $1092 \mathrm{~cm}^{-1} ;{ }^{1} \mathrm{H}$ NMR $\left(500 \mathrm{MHz}, \mathrm{CDCl}_{3}\right) \delta 7.64-7.60(\mathrm{~m}$, $4 \mathrm{H}), 7.36-7.34(\mathrm{~m}, 1 \mathrm{H}), 7.26-7.23(\mathrm{~m}, 4 \mathrm{H}), 7.19-7.17(\mathrm{~m}, 2 \mathrm{H}), 7.07-7.05(\mathrm{~m}, 1 \mathrm{H}), 6.86(\mathrm{~s}$, $1 \mathrm{H}), 6.41(\mathrm{~s}, 1 \mathrm{H}), 5.91(\mathrm{t}, J=7.9 \mathrm{~Hz}, 1 \mathrm{H}), 3.09$ (dd, $J=7.9,18.5 \mathrm{~Hz}, 1 \mathrm{H}), 2.59$ (dd, $J=7.9$, $18.5 \mathrm{~Hz}, 1 \mathrm{H}), 2.40(\mathrm{~s}, 3 \mathrm{H}), 2.35(\mathrm{~s}, 3 \mathrm{H}) ;{ }^{13} \mathrm{C} \mathrm{NMR}\left(125 \mathrm{MHz}, \mathrm{CDCl}_{3}\right) \delta 198.2,146.1,144.3$, $138.9,136.2$, 135.6, 133.8, 130.4, 130.3, 129.8, 129.5, 129.3, 127.5, 127.3, 126.8, 126.1, 
106.4, 76.4, 41.7, 21.6, 21.5; HRMS (ESI-TOF) calcd for $\mathrm{C}_{25} \mathrm{H}_{24} \mathrm{NO}_{4} \mathrm{~S}[\mathrm{M}+\mathrm{H}]^{+} \mathrm{m} / \mathrm{z}$ 434.1426, found 434.1427 .

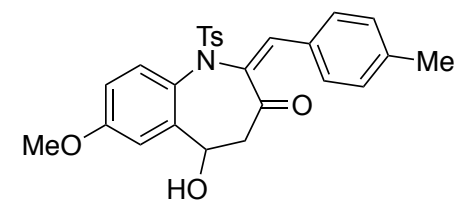

Following the general procedure, 3p $(86.1 \mathrm{mg}, 93 \%)$ was obtained as a yellow oil from $N$-(2-formyl-4-methoxyphenyl)-4-methylbenzenesulfonamide $1 \mathbf{b}(61.0 \mathrm{mg}, 0.2 \mathrm{mmol}) ; R_{\mathrm{f}}=$ 0.40 (hexane/EtOAc, 3:1); FCC (hexanes/ethyl acetate 10:1 to 3:1); IR (film) $v_{\max } 3271,2985$, 1726, 1500, 1320, 1251, 1194, 1158, $1092 \mathrm{~cm}^{-1} ;{ }^{1} \mathrm{H}$ NMR $\left(500 \mathrm{MHz}, \mathrm{CDCl}_{3}\right) \delta 7.66-7.64(\mathrm{~m}$, $2 \mathrm{H}), 7.60-7.58(\mathrm{~m}, 2 \mathrm{H}), 7.27-7.25(\mathrm{~m}, 2 \mathrm{H}), 7.17-7.16(\mathrm{~m}, 1 \mathrm{H}), 6.933-6.927(\mathrm{~m}, 1 \mathrm{H})$, 6.74-6.68 (m, 2H), $6.41(\mathrm{~s}, 1 \mathrm{H}), 6.38(\mathrm{~s}, 1 \mathrm{H}), 6.01(\mathrm{t}, J=7.9 \mathrm{~Hz}, 1 \mathrm{H}), 3.71(\mathrm{~s}, 3 \mathrm{H}), 3.16(\mathrm{dd}$, $J=7.9,18.5 \mathrm{~Hz}, 1 \mathrm{H}), 2.55(\mathrm{dd}, J=7.9,18.5 \mathrm{~Hz}, 1 \mathrm{H}), 2.40(\mathrm{~s}, 3 \mathrm{H}), 2.35(\mathrm{~s}, 3 \mathrm{H}) ;{ }^{13} \mathrm{C} \mathrm{NMR}$ $\left(125 \mathrm{MHz}, \mathrm{CDCl}_{3}\right) \delta 198.7,159.5,146.4,144.2,140.5,138.7,136.1,130.6,130.3,129.8$, $129.5,129.4,127.5,125.3,114.1,111.7,105.8,75.7,55.5,42.4,21.6,21.5$; HRMS (ESI-TOF) calcd for $\mathrm{C}_{26} \mathrm{H}_{26} \mathrm{NO}_{5} \mathrm{~S}[\mathrm{M}+\mathrm{H}]^{+} m / z$ 464.1532, found 464.1538.

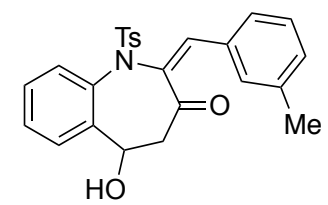

Following the general procedure, $\mathbf{3 q}(73.6 \mathrm{mg}, 85 \%)$ was obtained as a colorless oil from $N$-(2-formylphenyl)-4-methylbenzenesulfonamide $\mathbf{1 a}(55.0 \mathrm{mg}, 0.2 \mathrm{mmol}) ; R_{\mathrm{f}}=0.35$ (hexane/EtOAc, 3:1); FCC (hexanes/ethyl acetate 10:1 to 3:1); IR (film) $v_{\max } 3258,2930$, 1728, 1600, 1338, 1251, 1167, $1095 \mathrm{~cm}^{-1} ;{ }^{1} \mathrm{H}$ NMR (500 MHz, $\left.\mathrm{CDCl}_{3}\right) \delta 7.61-7.57$ (m, 3H), $7.52(\mathrm{~s}, 1 \mathrm{H}), 7.37-7.35(\mathrm{~m}, 1 \mathrm{H}), 7.28-7.22(\mathrm{~m}, 5 \mathrm{H}), 7.13-7.11(\mathrm{~m}, 1 \mathrm{H}), 7.06-7.04(\mathrm{~m}, 1 \mathrm{H})$, $6.84(\mathrm{~s}, 1 \mathrm{H}), 6.39$ (s, 1H), 5.93 (t, $J=7.9 \mathrm{~Hz}, 1 \mathrm{H}), 3.10$ (dd, $J=7.9,18.5 \mathrm{~Hz}, 1 \mathrm{H}), 2.61$ (dd, $J$ $=7.9,18.5 \mathrm{~Hz}, 1 \mathrm{H}), 2.40(\mathrm{~s}, 3 \mathrm{H}), 2.36(\mathrm{~s}, 3 \mathrm{H}) ;{ }^{13} \mathrm{C} \mathrm{NMR}\left(125 \mathrm{MHz}, \mathrm{CDCl}_{3}\right) \delta 198.3,146.4$, $144.3,138.3,136.2,135.7,133.8,133.2,130.9,129.8,129.5,129.4,128.6,127.6,127.4$, 127.3, 126.8, 126.2, 106.3, 76.4, 41.7, 21.6, 21.5; HRMS (ESI-TOF) calcd for $\mathrm{C}_{25} \mathrm{H}_{24} \mathrm{NO}_{4} \mathrm{~S}$ $[\mathrm{M}+\mathrm{H}]^{+} \mathrm{m} / \mathrm{z}$ 434.1426, found 434.1430 .

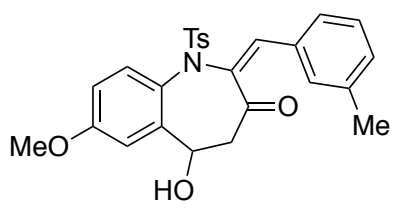

Following the general procedure, $3 \mathbf{r}(75.0 \mathrm{mg}, 81 \%)$ was obtained as a yellow oil from $N$-(2-formyl-4-methoxyphenyl)-4-methylbenzenesulfonamide 1 b $(61.0 \mathrm{mg}, 0.2 \mathrm{mmol}) ; R_{\mathrm{f}}=$ 0.35 (hexane/EtOAc, 3:1); FCC (hexanes/ethyl acetate 10:1 to 3:1); IR (film) $v_{\max } 3250,3006$, 2989, 1720, 1632, 1502, 1160, $1078 \mathrm{~cm}^{-1}$; ${ }^{1} \mathrm{H} \mathrm{NMR}\left(500 \mathrm{MHz}, \mathrm{CDCl}_{3}\right) \delta 7.60-7.53(\mathrm{~m}, 4 \mathrm{H})$, 7.27-7.23 (m, 3H), 7.11-7.10 (m, 1H), 6.94-6.93 (m, 1H), 6.73-6.67 (m, 2H), $6.49(\mathrm{~s}, 1 \mathrm{H})$, $6.36(\mathrm{~s}, 1 \mathrm{H}), 6.04(\mathrm{t}, J=7.9 \mathrm{~Hz}, 1 \mathrm{H}), 3.71(\mathrm{~s}, 3 \mathrm{H}), 3.18(\mathrm{dd}, J=7.9,18.5 \mathrm{~Hz}, 1 \mathrm{H}), 2.56(\mathrm{dd}, J$ $=7.9,18.5 \mathrm{~Hz}, 1 \mathrm{H}), 2.40(\mathrm{~s}, 3 \mathrm{H}), 2.35(\mathrm{~s}, 3 \mathrm{H}) ;{ }^{13} \mathrm{C} \mathrm{NMR}\left(125 \mathrm{MHz}, \mathrm{CDCl}_{3}\right) \delta 198.8,159.5$, $146.8,144.2,140.6,138.2,136.1,133.4,130.9,129.8,129.5,129.4,128.6,127.5,127.4$, 125.2, 114.1, 111.7, 105.8, 75.8, 55.5, 42.3, 21.6, 21.5; HRMS (ESI-TOF) calcd for 
$\mathrm{C}_{26} \mathrm{H}_{26} \mathrm{NO}_{5} \mathrm{~S}[\mathrm{M}+\mathrm{H}]^{+} m / z$ 464.1532, found 464.1538.

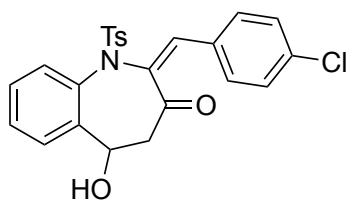

Following the general procedure, $3 \mathbf{s}(68.0 \mathrm{mg}, 75 \%)$ was obtained as a pale solid from $N$-(2-formylphenyl)-4-methylbenzenesulfonamide 1a (55.0 mg, $0.2 \mathrm{mmol}$ ); m.p. $160-162{ }^{\circ} \mathrm{C}$; $R_{\mathrm{f}}=0.42$ (hexane/EtOAc, 3:1); FCC (hexanes/ethyl acetate $10: 1$ to $3: 1$ ); IR (film) $v_{\max } 3254$, $3001,2985,1744,1491,1334,1160,1092 \mathrm{~cm}^{-1} ;{ }^{1} \mathrm{H}$ NMR $\left(500 \mathrm{MHz}, \mathrm{CDCl}_{3}\right) \delta 7.67-7.66(\mathrm{~m}$, 2H), 7.60-7.59 (m, 2H), 7.38-7.37 (m, 1H), 7.33-7.27 (m, 4H), 7.25-7.23 (m, 1H), 6.94-6.93 (m, 1H), $6.61(\mathrm{~s}, 1 \mathrm{H}), 6.36(\mathrm{~s}, 1 \mathrm{H}), 6.05(\mathrm{t}, J=7.9 \mathrm{~Hz}, 1 \mathrm{H}), 3.17(\mathrm{dd}, J=7.9,18.7 \mathrm{~Hz}, 1 \mathrm{H})$, $2.62(\mathrm{dd}, J=7.9,18.7 \mathrm{~Hz}, 1 \mathrm{H}), 2.41(\mathrm{~s}, 3 \mathrm{H}) ;{ }^{13} \mathrm{C} \mathrm{NMR}\left(125 \mathrm{MHz}, \mathrm{CDCl}_{3}\right) \delta 198.2,146.9$, $144.4,136.5,136.0,134.1,133.5,131.9,131.4,129.8,129.4,128.9,127.9,127.4,126.8$, 126.6, 104.5, 76.5, 41.9, 21.6; HRMS (ESI-TOF) calcd for $\mathrm{C}_{24} \mathrm{H}_{21} \mathrm{ClNO}_{4} \mathrm{~S}[\mathrm{M}+\mathrm{H}]^{+} \mathrm{m} / z$ 454.0880 , found 454.0882 .

\section{Synthesis of the product 31 on $1 \mathrm{mmol}$ scale}

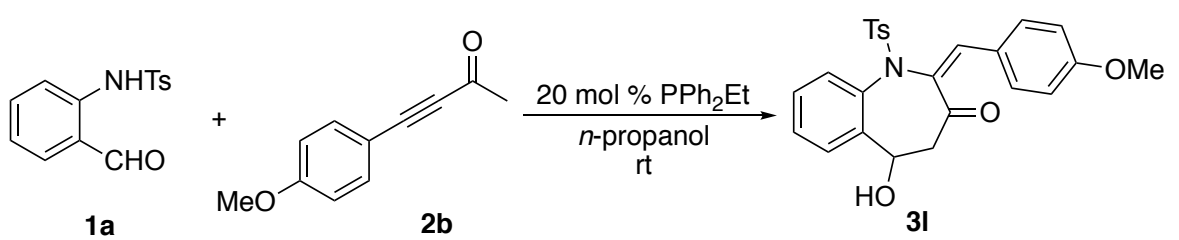

The $o$-( $N$-tosyl)benzaldehyde (1a) $(275 \mathrm{mg}, 1.0 \mathrm{mmol})$, the ynone (2b) $(261 \mathrm{mg}, 1.5 \mathrm{mmol}$, 1.5 equiv), $\mathrm{Ph}_{2} \mathrm{EtP}$ (42.8 mg, $0.2 \mathrm{mmol}, 0.2$ equiv), and $n$-propanol $(10 \mathrm{~mL}$ ) were added sequentially to a flame-dried flask $(50 \mathrm{~mL})$ and then the reaction mixture was stirred at room temperature until the starting material 1 was consumed. The solvent was evaporated under reduced pressure and the crude product was purified through silica gel flash column chromatography (hexanes/ethyl acetate $10: 1$ to $3: 1$ ) to yield the desired product $\mathbf{3 l}$ (372 $\mathrm{mg}$, $83 \%$ yield).

\section{Characterization Data for Compound 4}

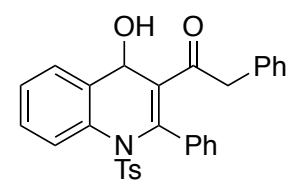

$4 \quad(91.1 \quad \mathrm{mg}, \quad 92 \%)$ was obtained as a yellow oil from $N$-(2-formylphenyl)-4-methylbenzenesulfonamide $\mathbf{1 a}(55.0 \mathrm{mg}, 0.2 \mathrm{mmol}) ; \quad R_{\mathrm{f}}=0.52$ (hexane/EtOAc, 3:1); FCC (hexanes/ethyl acetate 10:1 to 3:1); IR (film) $v_{\max } 3327,2989$, 1729, 1494, 1336, 1162, $1092 \mathrm{~cm}^{-1} ;{ }^{1} \mathrm{H}$ NMR (500 MHz, $\left.\mathrm{CDCl}_{3}\right) \delta 7.78-7.76(\mathrm{~m}, 2 \mathrm{H})$, 7.53-7.51 (m, 2H), 7.47-7.41 (m, 3H), 7.39-7.33 (m, 5H), 7.28-7.27 (m, 1H), 7.25-7.22 (m, 1H), 7.20-7.18 (m, 2H), 6.97-6.95 (m, 2H), $6.56(\mathrm{~s}, 1 \mathrm{H}), 6.30(\mathrm{~s}, 1 \mathrm{H}), 5.22(\mathrm{~d}, J=9.7 \mathrm{~Hz}$, $1 \mathrm{H}), 3.51(\mathrm{~d}, J=9.7 \mathrm{~Hz}, 1 \mathrm{H}), 2.23(\mathrm{~s}, 3 \mathrm{H}) ;{ }^{13} \mathrm{C} \mathrm{NMR}\left(125 \mathrm{MHz}, \mathrm{CDCl}_{3}\right) \delta 197.6,146.2$, $144.2,136.8,134.2,133.8,133.0,131.5,130.5,129.9,129.8,129.5,129.1,128.9,128.7$, 
128.5, 126.9 (2C), 126.3, 108.1, 84.4, 58.9, 21.4; HRMS (ESI-TOF) calcd for $\mathrm{C}_{30} \mathrm{H}_{26} \mathrm{NO}_{4} \mathrm{~S}$ $[\mathrm{M}+\mathrm{H}]^{+} \mathrm{m} / \mathrm{z}$ 496.1583, found 496.1590.

\section{Characterization Data for Compounds 5 and 6.}

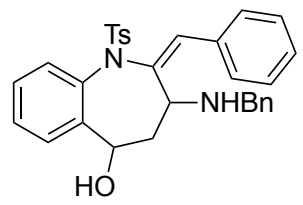

$\mathrm{TiCl}_{4}(0.5 \mathrm{~mL}, 1.0 \mathrm{~mol} / \mathrm{L}$ in DCM$)$ was slowly added to a solution of $\mathbf{3 a}(248 \mathrm{mg}, 0.5 \mathrm{mmol})$ and $\mathrm{BnNH}_{2}\left(80 \mathrm{mg}, 0.75 \mathrm{mmol}, 1.5\right.$ equiv) in $\mathrm{CH}_{2} \mathrm{Cl}_{2}(20 \mathrm{~mL})$ and then the mixture was stirred at room temperature until 3a had been consumed (monitored using TLC). The $\mathrm{CH}_{2} \mathrm{Cl}_{2}$ was evaporated and the residue dissolved in $\mathrm{MeOH}(20 \mathrm{~mL}) . \mathrm{NaBH}_{3} \mathrm{CN}(47 \mathrm{mg}, 0.75 \mathrm{mmol}$, 1.5 equiv) was added and then the mixture was stirred at room temperature until all of the intermediate had been consumed. The solution was concentrated under reduced pressure and the residue purified by silica gel flash column chromatography (hexanes/ethyl acetate 10:1 to 2:1) to yield the amine 5 .

5: Yellow oil (217 mg, 85\% yield); $R_{\mathrm{f}}=0.80$ (hexane/EtOAc, 1:1); IR (film) $v_{\max } 3287,3054$, 1674, 1493, 1330, 1194, 1157, $1092 \mathrm{~cm}^{-1} ;{ }^{1} \mathrm{H}$ NMR (500 MHz, $\left.\mathrm{CDCl}_{3}\right) \delta 7.58-7.53(\mathrm{~m}, 4 \mathrm{H})$, 7.42-7.37 (m, 5H), 7.31-7.28 (m, 3H), 7.24-7.22 (m, 2H), 7.17-7.08 (m, 4H), $5.60(\mathrm{~s}, 1 \mathrm{H})$, $5.34(\mathrm{t}, J=7.9 \mathrm{~Hz}, 1 \mathrm{H}), 4.06-4.02(\mathrm{~m}, 2 \mathrm{H}), 3.88(\mathrm{~d}, J=13.8 \mathrm{~Hz}, 1 \mathrm{H}), 2.71-2.66(\mathrm{~m}, 1 \mathrm{H})$, $2.32(\mathrm{~s}, 3 \mathrm{H}), 1.88-1.82(\mathrm{~m}, 1 \mathrm{H}) ;{ }^{13} \mathrm{C} \mathrm{NMR}\left(125 \mathrm{MHz}, \mathrm{CDCl}_{3}\right) \delta 156.8,143.5,139.4,137.1$, 135.7, 135.2, 131.3, 129.6, 129.3, 128.6, 128.5, 128.4, 128.2, 127.9, 127.3 (2C), 125.6, 125.0, $122.8,99.7,81.7,59.4,51.0,37.9,21.5$; HRMS (ESI-TOF) calcd for $\mathrm{C}_{31} \mathrm{H}_{31} \mathrm{~N}_{2} \mathrm{O}_{3} \mathrm{~S}[\mathrm{M}+\mathrm{H}]^{+}$ $\mathrm{m} / \mathrm{z} 511.2055$, found 511.2060.

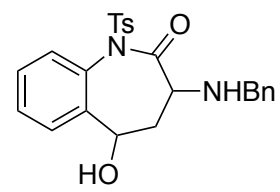

A solution of the amine $5(102 \mathrm{mg}, 0.2 \mathrm{mmol})$ in $\mathrm{MeOH} / \mathrm{CH}_{2} \mathrm{Cl}_{2}(3: 1,20 \mathrm{~mL})$ was cooled to $-78{ }^{\circ} \mathrm{C}$. Ozone was passed through the solution until the starting material had been consumed (TLC). The mixture was warmed to room temperature and the excess ozone was removed using a stream of $\mathrm{N}_{2}$. DMS (2 $\mathrm{mL}$ ) was added and then the solution was stirred at room temperature overnight. The solution was concentrated under reduced pressure and the residue purified by silica gel flash column chromatography (hexanes/ethyl acetate 10:1 to 1:1)to yield 6.

6: Colorless oil (514. mg, 62\% yield); $R_{\mathrm{f}}=0.50$ (hexane/EtOAc, 1:1); IR (film) $v_{\max } 3263$, $3047,1772,1325,1194,1157,1092 \mathrm{~cm}^{-1} ;{ }^{1} \mathrm{H}$ NMR (500 MHz, $\left.\mathrm{CDCl}_{3}\right) \delta 7.60-7.59(\mathrm{~m}, 2 \mathrm{H})$, 7.40-7.34 (m, 5H), 7.30-7.24 (m, 4H), 7.20-7.17 (m, 1H), 6.84-6.82 (m, 1H), $5.64(\mathrm{dd}, J=$ $5.5,11.0 \mathrm{~Hz}, 1 \mathrm{H}), 3.95(\mathrm{~d}, J=13.2 \mathrm{~Hz}, 1 \mathrm{H}), 3.90(\mathrm{~d}, J=13.2 \mathrm{~Hz}, 1 \mathrm{H}), 3.77$ (dd, $J=7.9,11.4$ $\mathrm{Hz}, 1 \mathrm{H}), 2.95-2.90(\mathrm{~m}, 1 \mathrm{H}), 2.41(\mathrm{~s}, 3 \mathrm{H}), 2.10-2.03(\mathrm{~m}, 1 \mathrm{H}) ;{ }^{13} \mathrm{C} \mathrm{NMR}\left(125 \mathrm{MHz}, \mathrm{CDCl}_{3}\right) \delta$ 176.3, 144.2, 138.9, 136.1, 134.9, 133.8, 129.8, 128.7, 128.2, 127.8, 127.5, 127.3, 126.6, 74.8, 57.1, 52.0, 37.8, 21.6; HRMS (ESI-TOF) calcd for $\mathrm{C}_{24} \mathrm{H}_{25} \mathrm{~N}_{2} \mathrm{O}_{4} \mathrm{~S}[\mathrm{M}+\mathrm{H}]^{+} m / z$ 437.1535, 
found 437.1531. 


\section{References}

(1) (a) Fonseca, M. H.; Eibler, E.; Zabelb, M.; Konig, B. Tetrahedron: Asymmetry 2003, 14, 1989. (b) Theeraladanon, C.; Arisawa, M.; Nishida, A.; Nakagawa, M. Tetrahedron 2004, 60, 3017. (c) Chernyak, D.; Chernyak, N.; Gevorgyan, V. Adv. Synth. Catal. 2010, 352, 961.

(2) (a) Cao, L.; Ding, J.; Gao, M.; Wang, Z.; Li, J.; Wu, A. Org. Lett. 2009, 11, 3810. (b) Schmidt, N. G.; Simon, R. C.; Kroutil, W. Adv. Synth. Catal. 2015, 357, 1815. (c) Li, Z.; Yu, H.; Liu, Y.; Zhou, L.; Sun, Z.; Guo, H. Adv. Synth. Catal. 2016, 358, 1880. (d) Sadamitsu, Y.; Komatsuki, K.; Saito, K.; Yamada, T. Org. Lett. 2017, 19, 3191. 


\section{NOESY Analysis of Compound E-3}
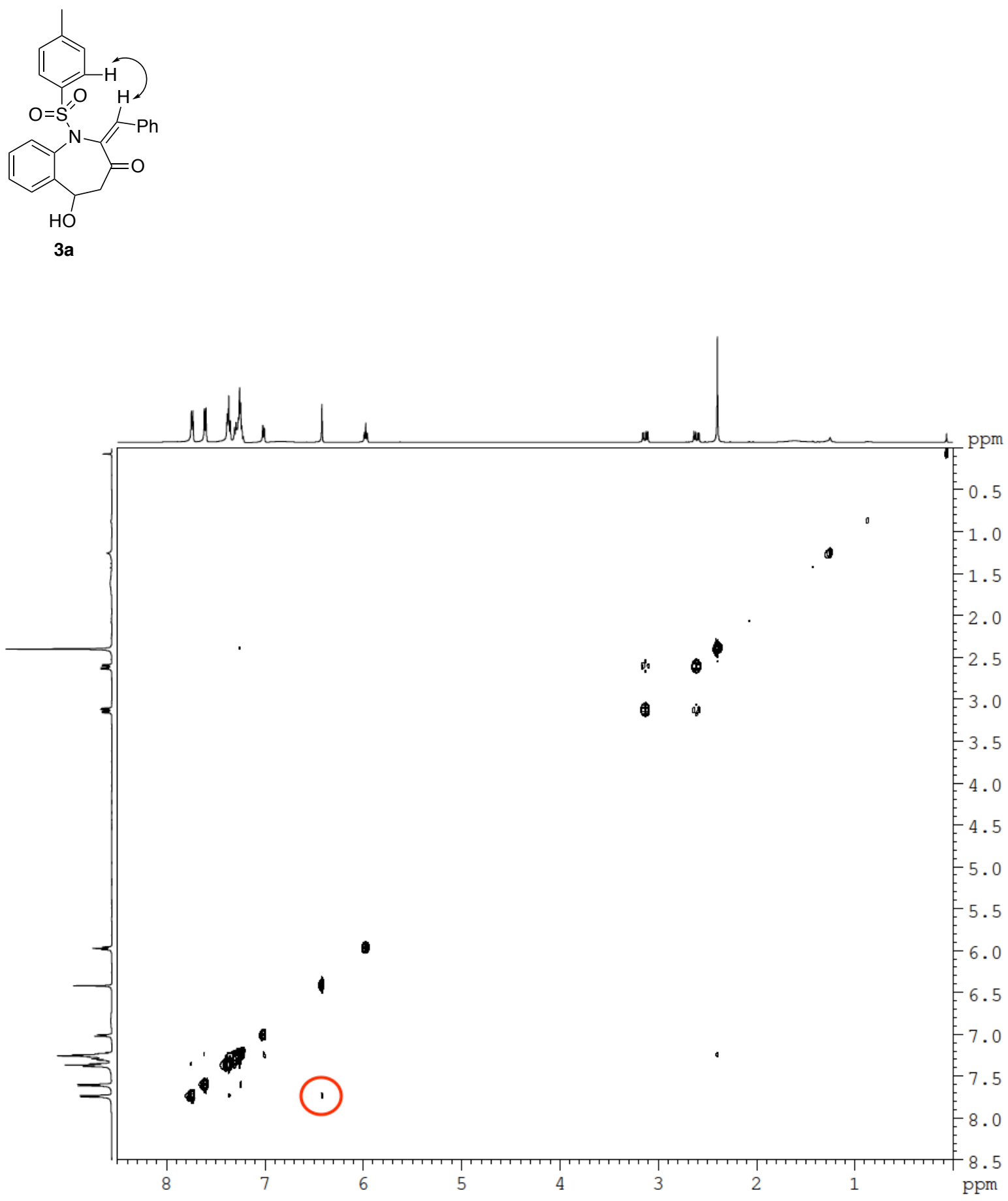

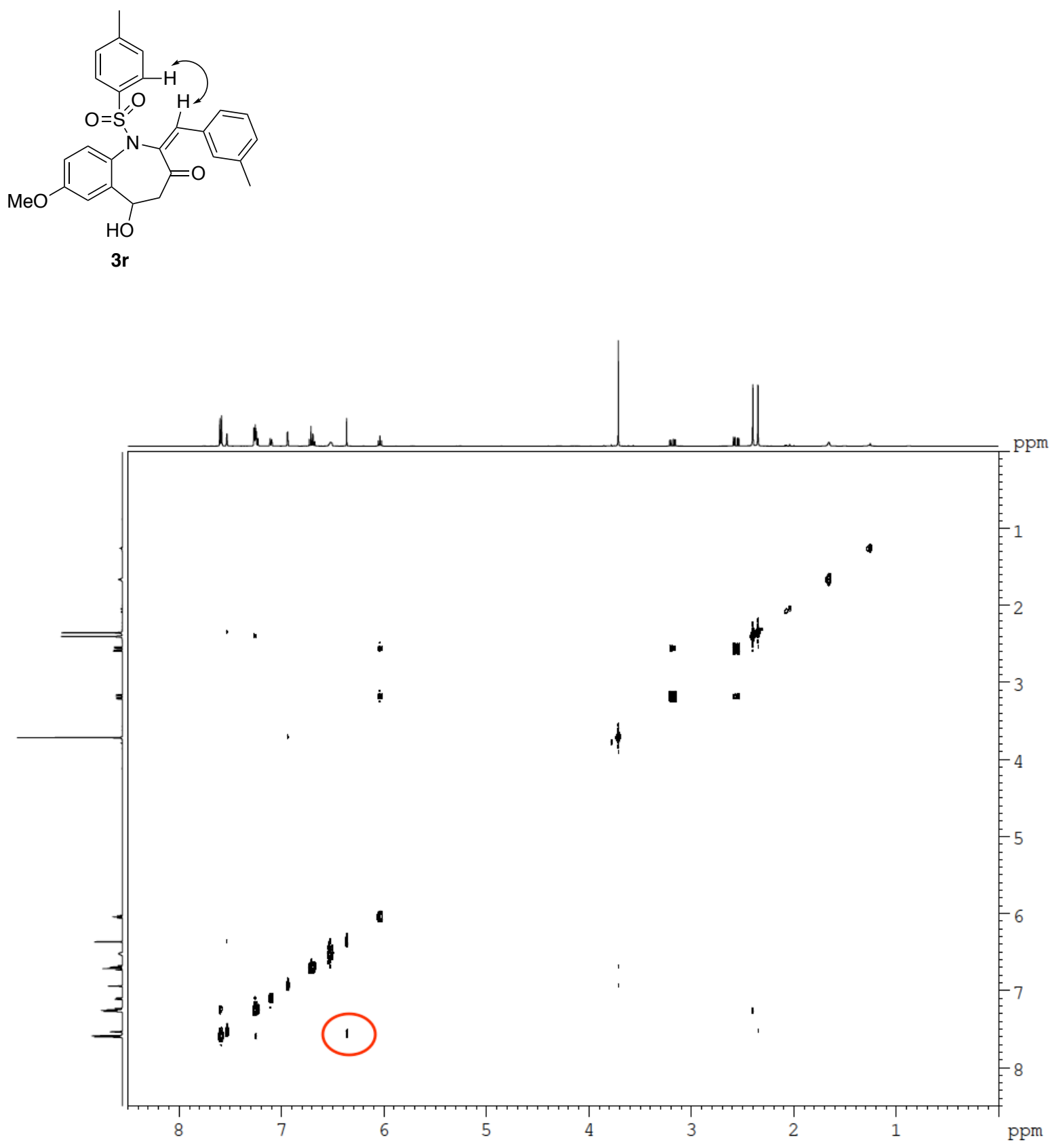


\section{6. ${ }^{1} \mathrm{H}$ NMR and ${ }^{13} \mathrm{C}$ NMR Spectra}

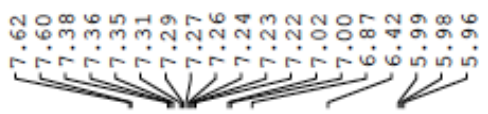

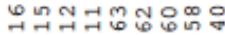

मिंलें $\dot{\sim} \dot{\sim} \dot{N}$<smiles>O=C1CC(O)c2ccccc2N([125I])/C1=C/c1ccccc1</smiles>

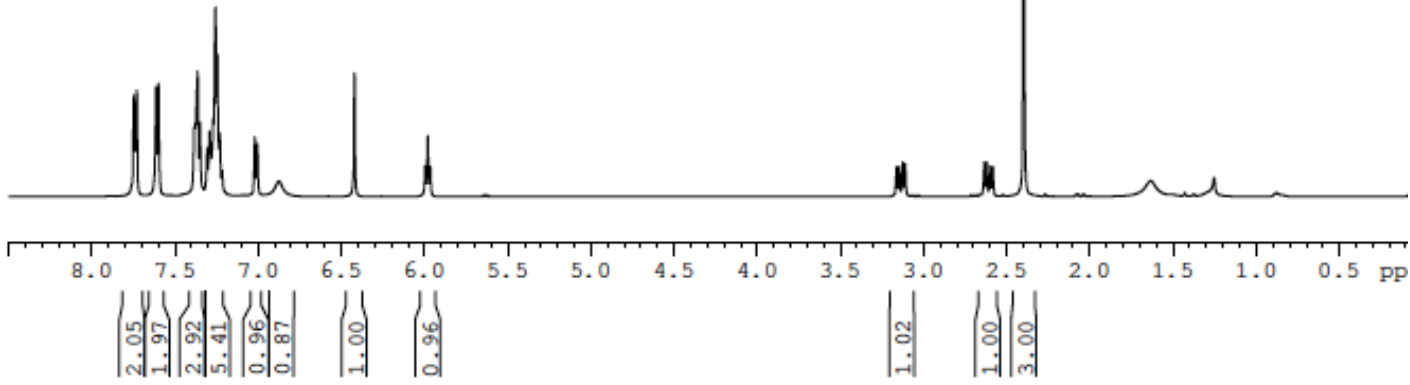

$\stackrel{\nabla}{\infty}$

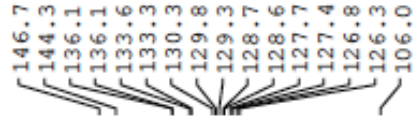

$\stackrel{\square}{\circ}$
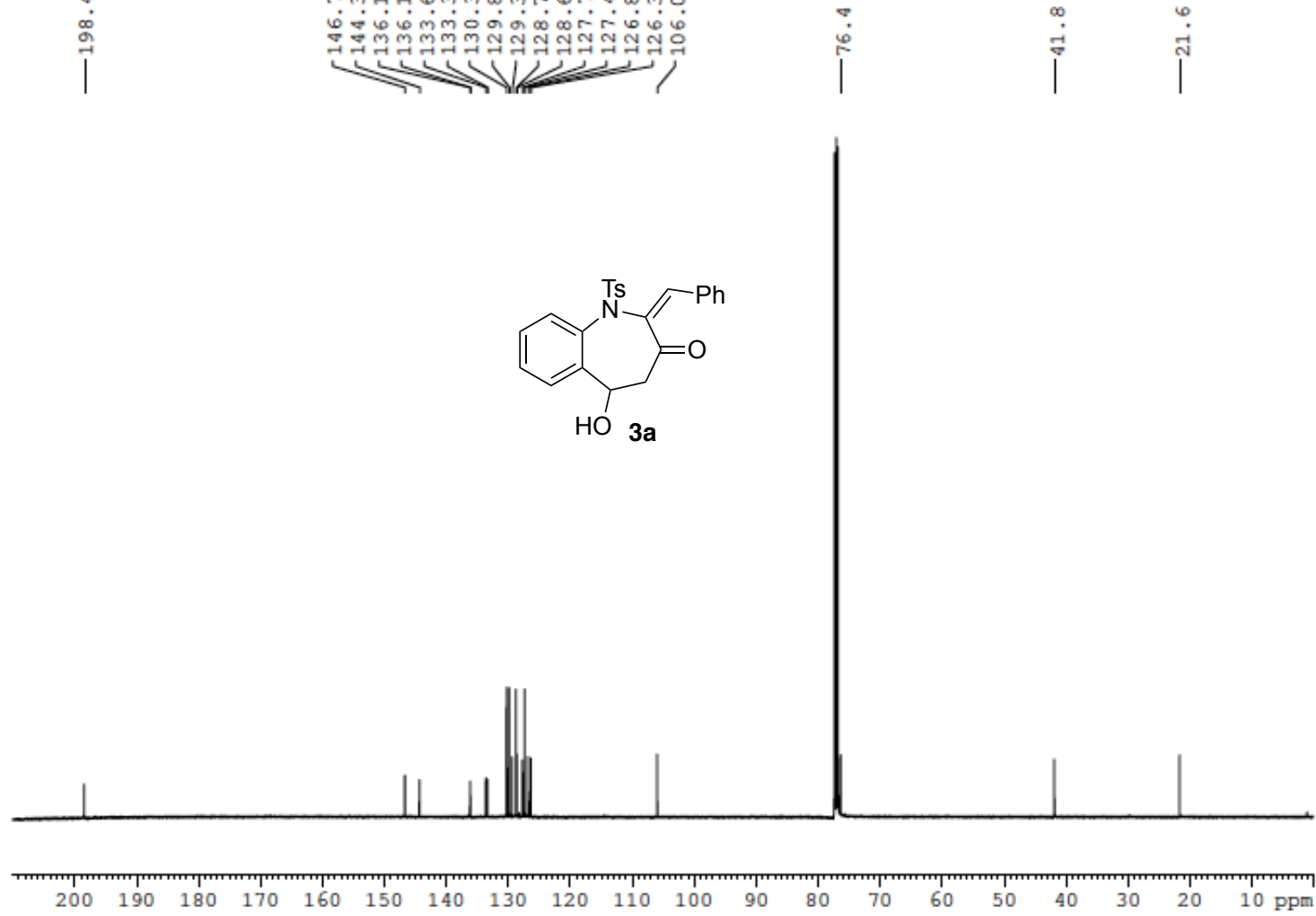
<smiles>COc1ccc2c(c1)C(O)CC(=O)/C2=C\c1ccccc1</smiles>

3b

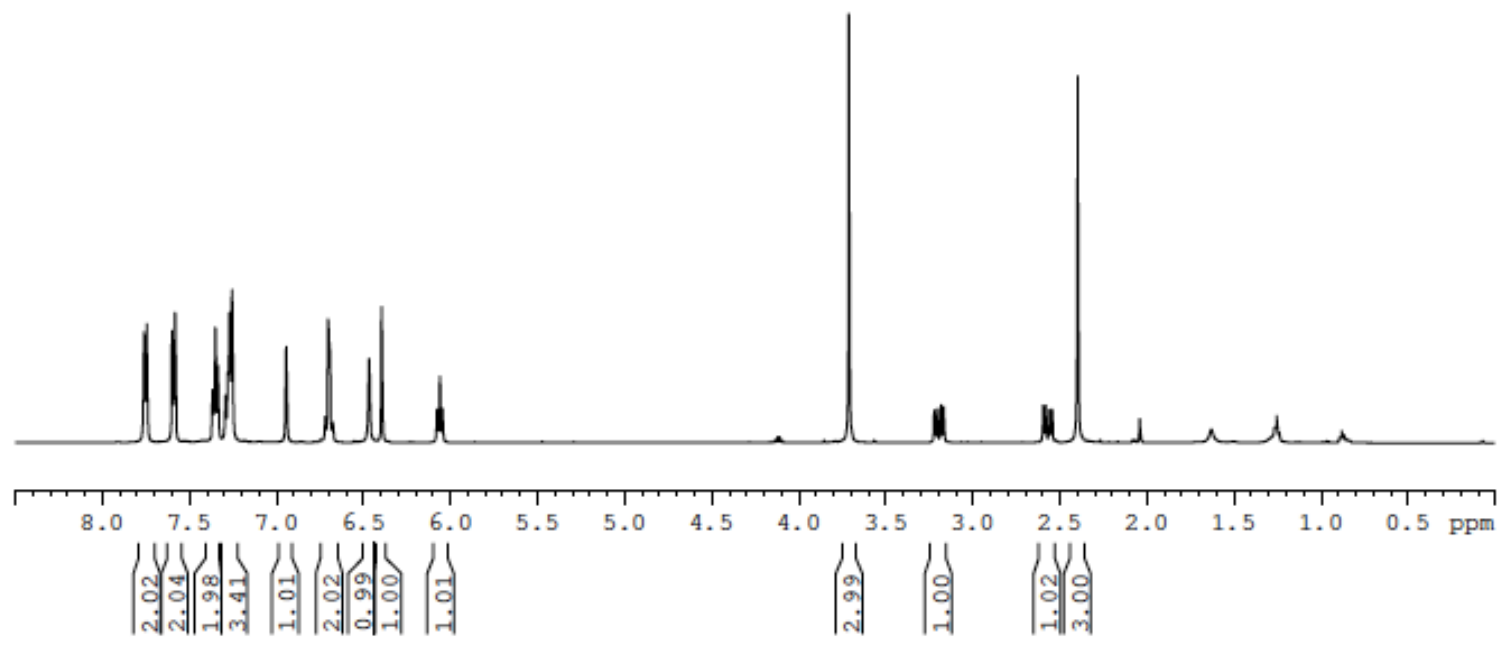

$\stackrel{\infty}{\infty} \stackrel{\infty}{\infty}$

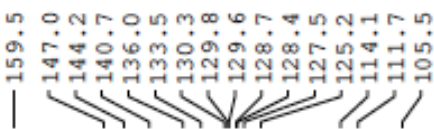

i
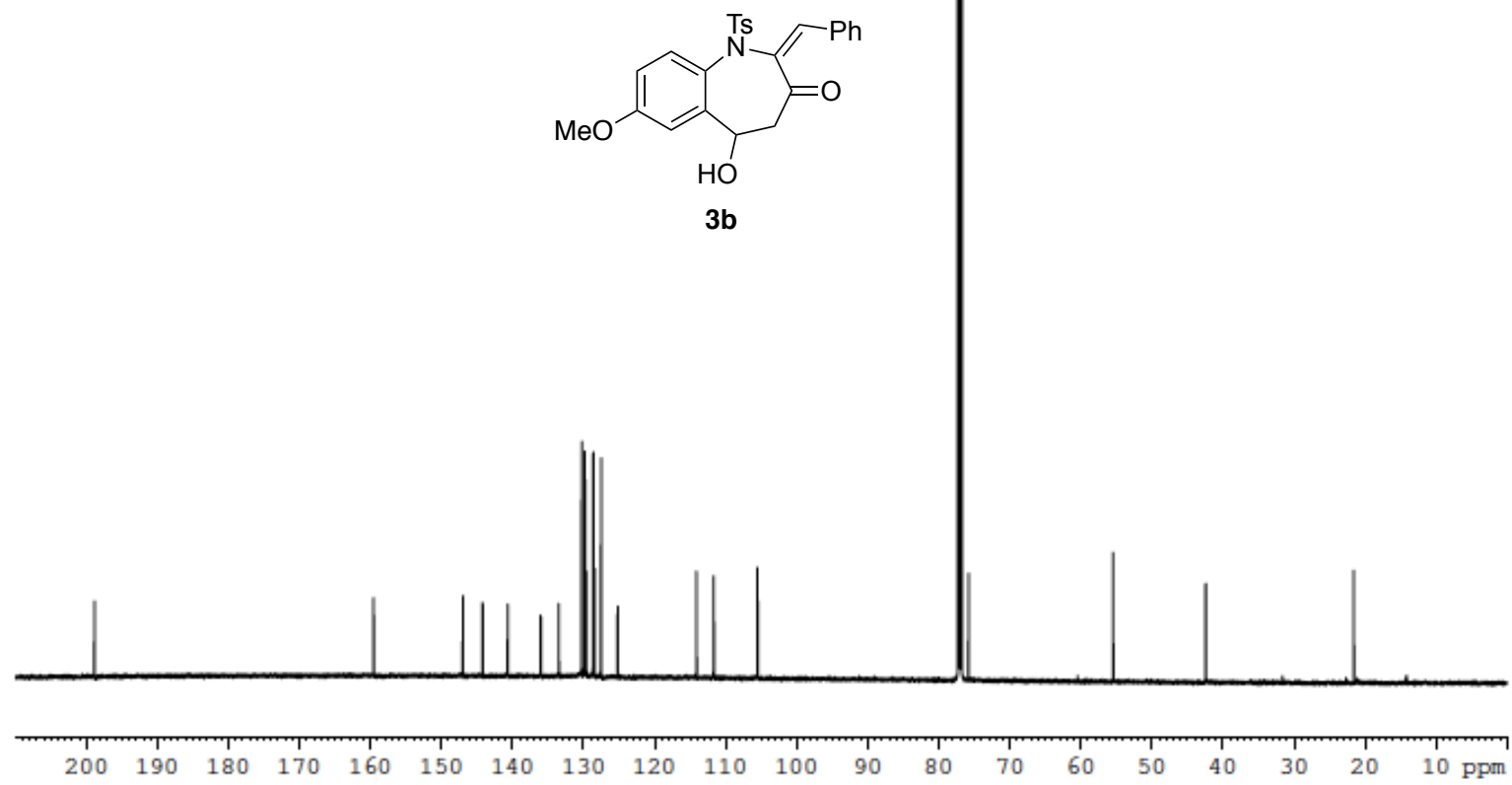

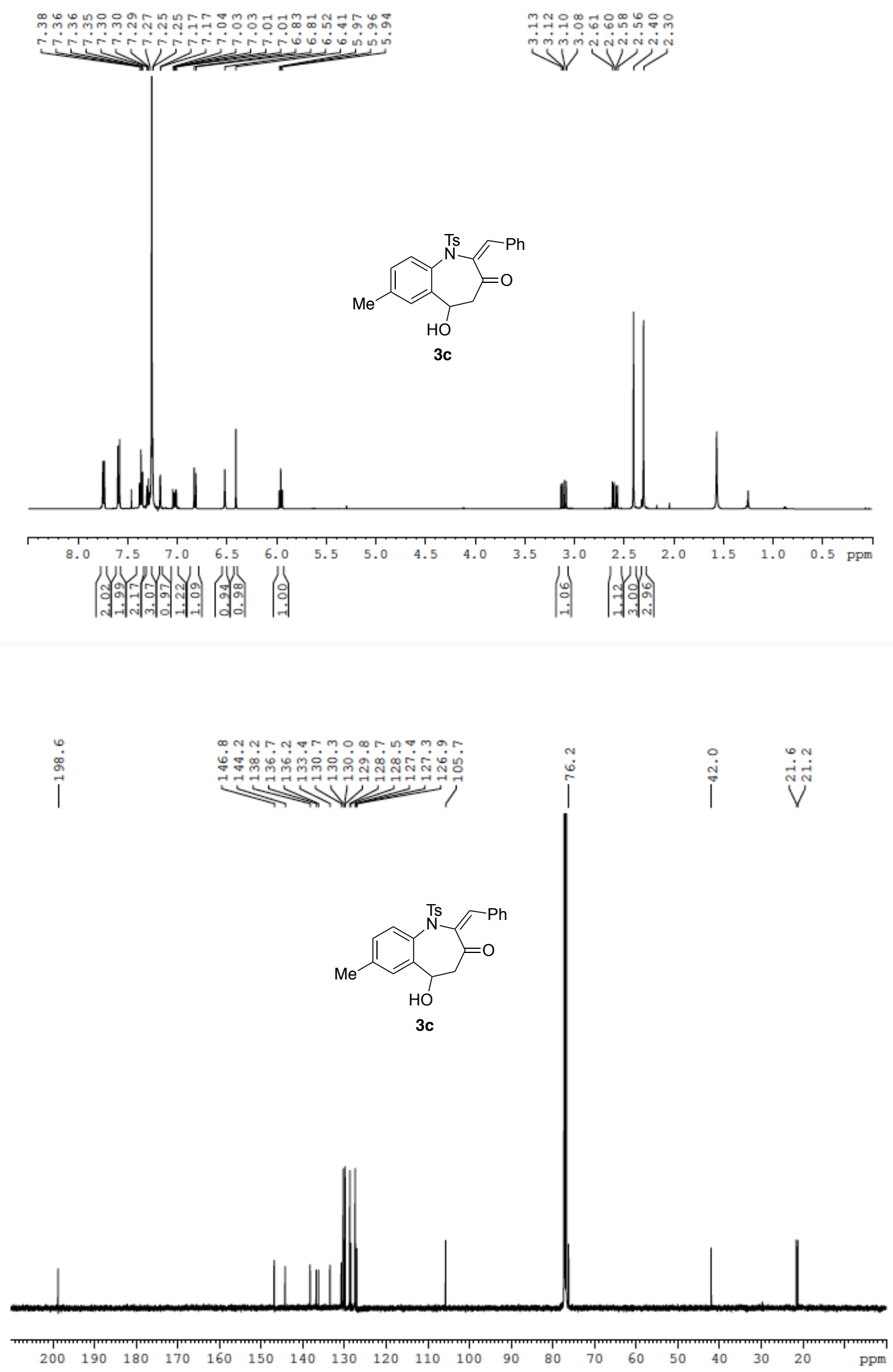

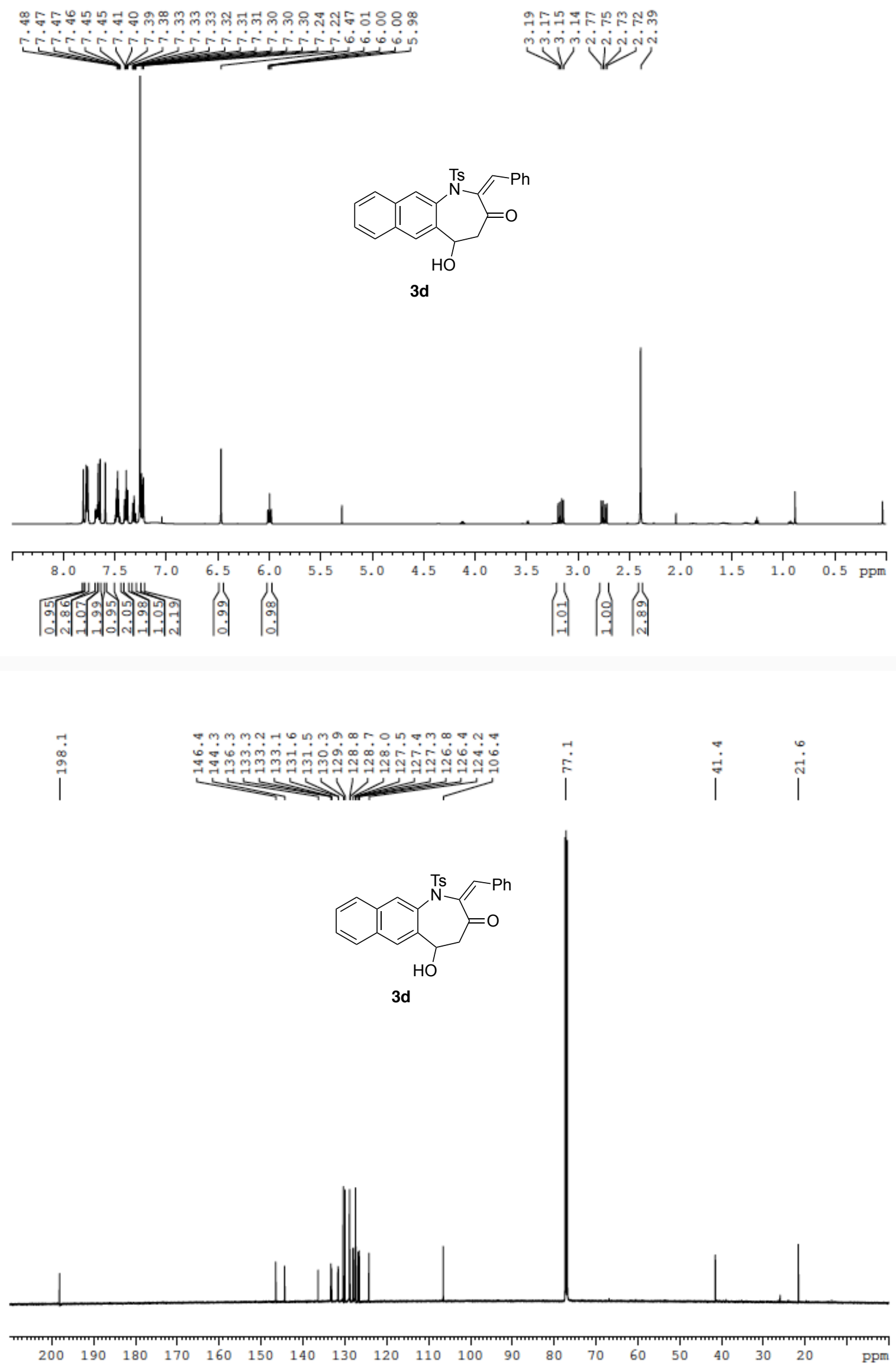

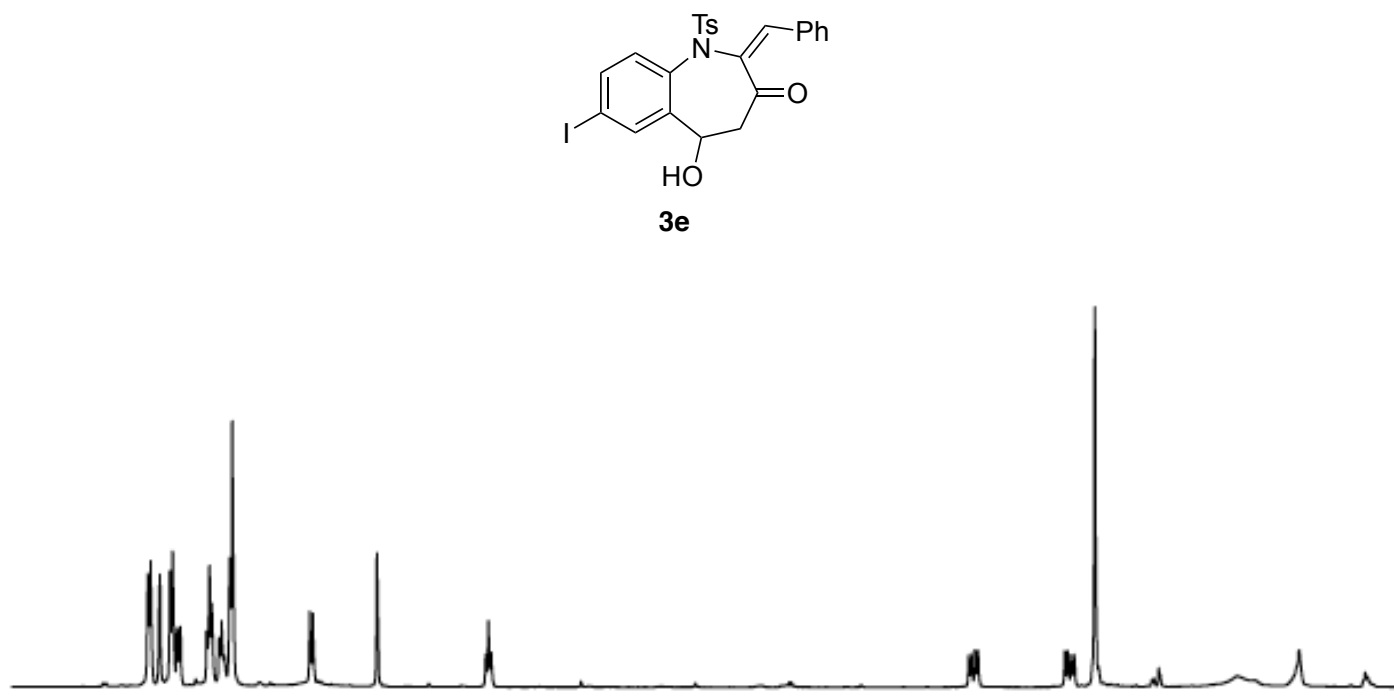

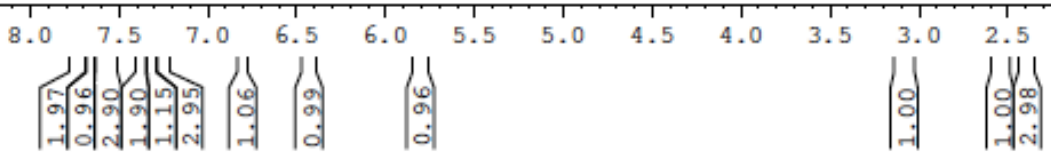

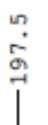
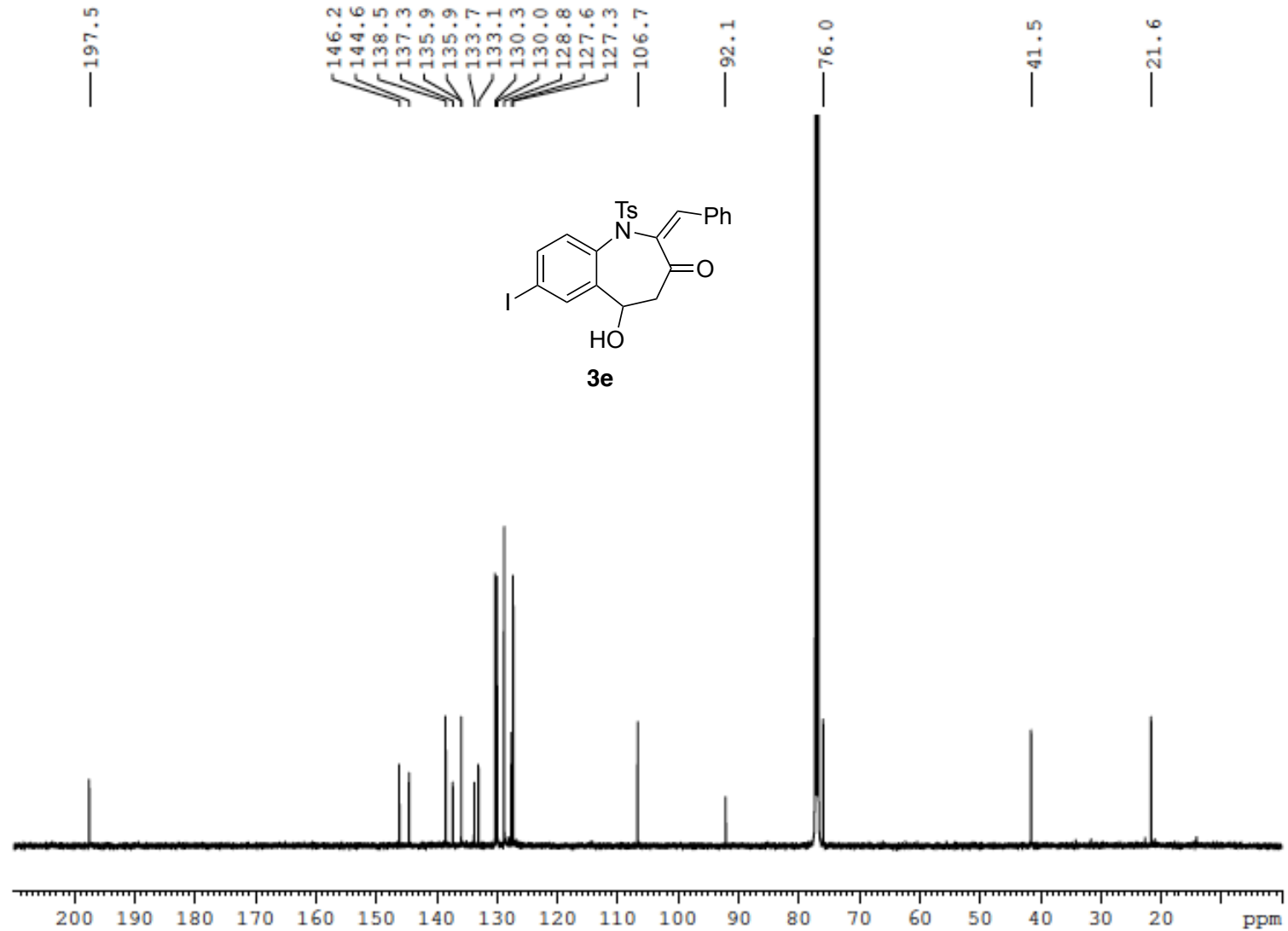

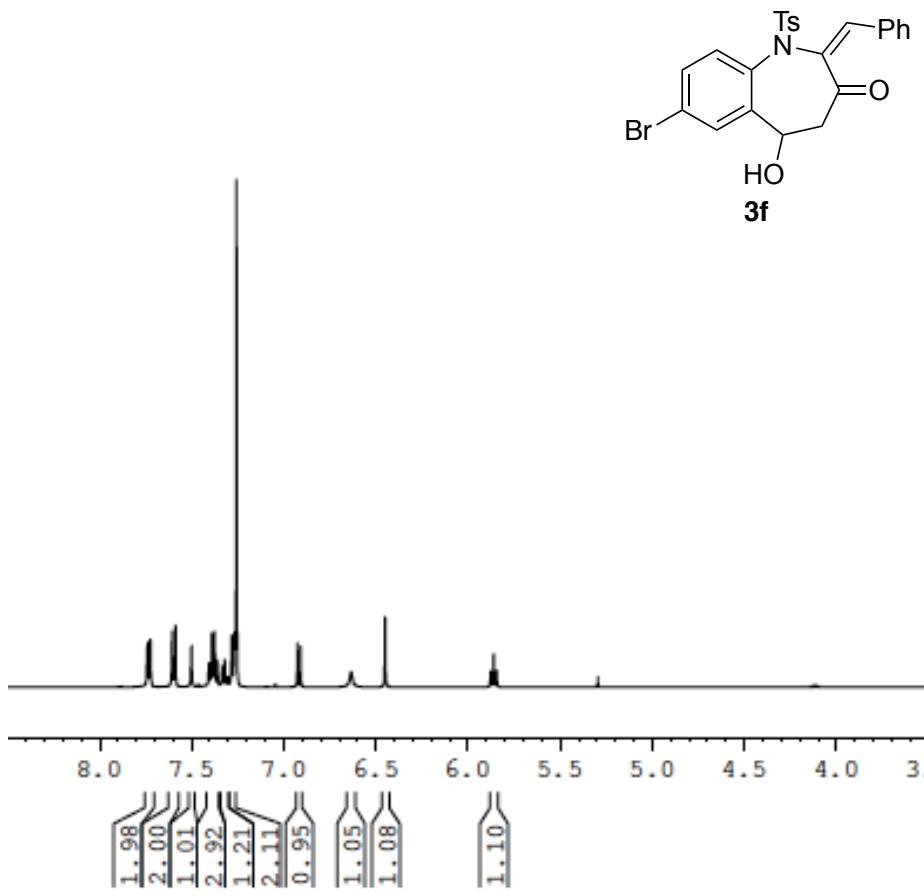

$3 f$
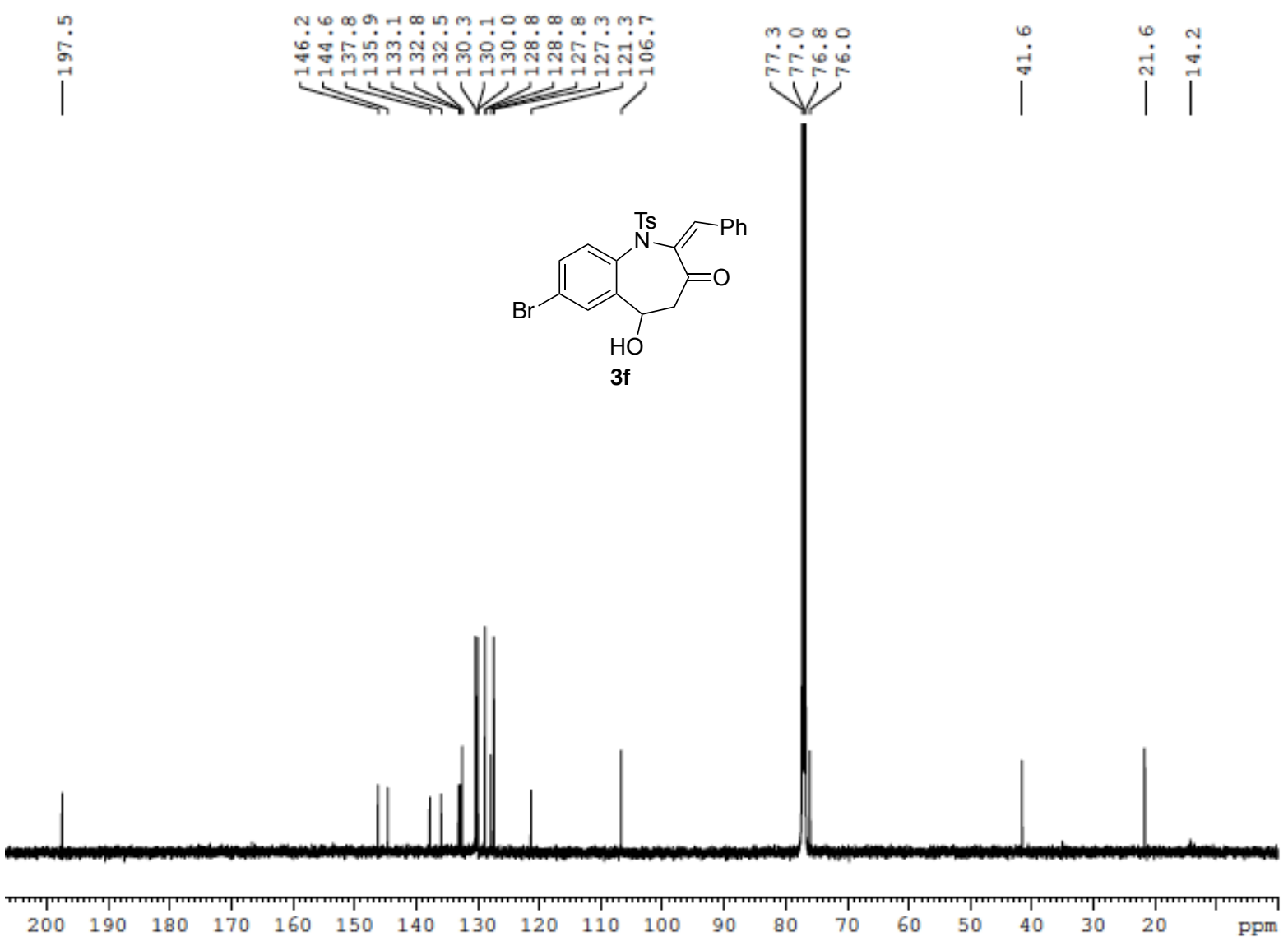

위 नौำ 

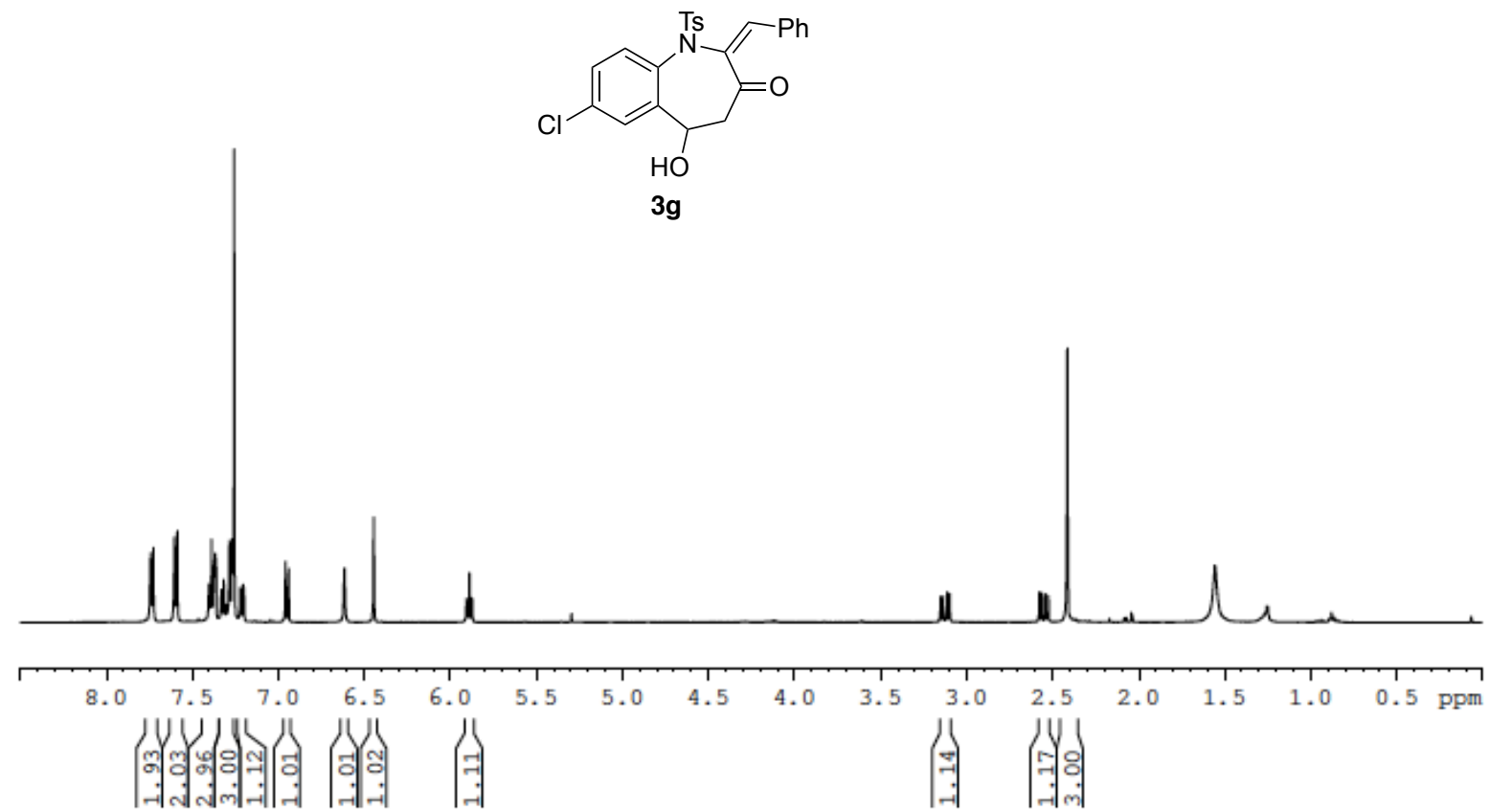

$\stackrel{\varphi}{\dot{1}}$
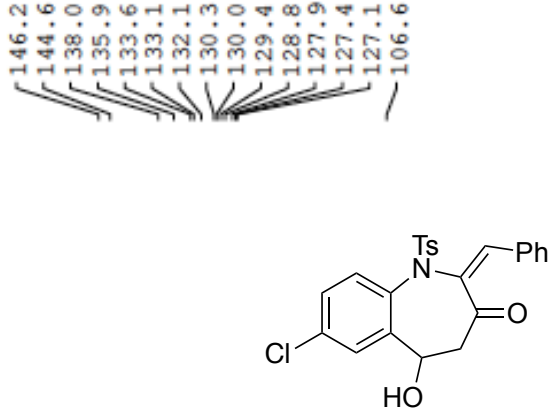

$3 \mathrm{~g}$

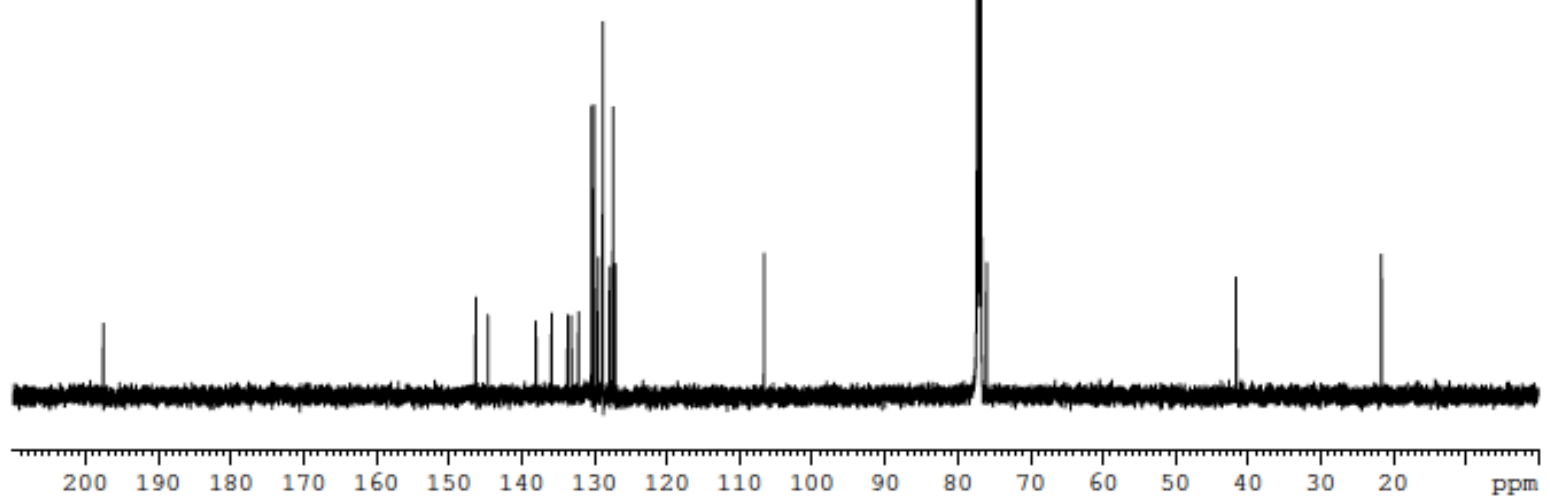



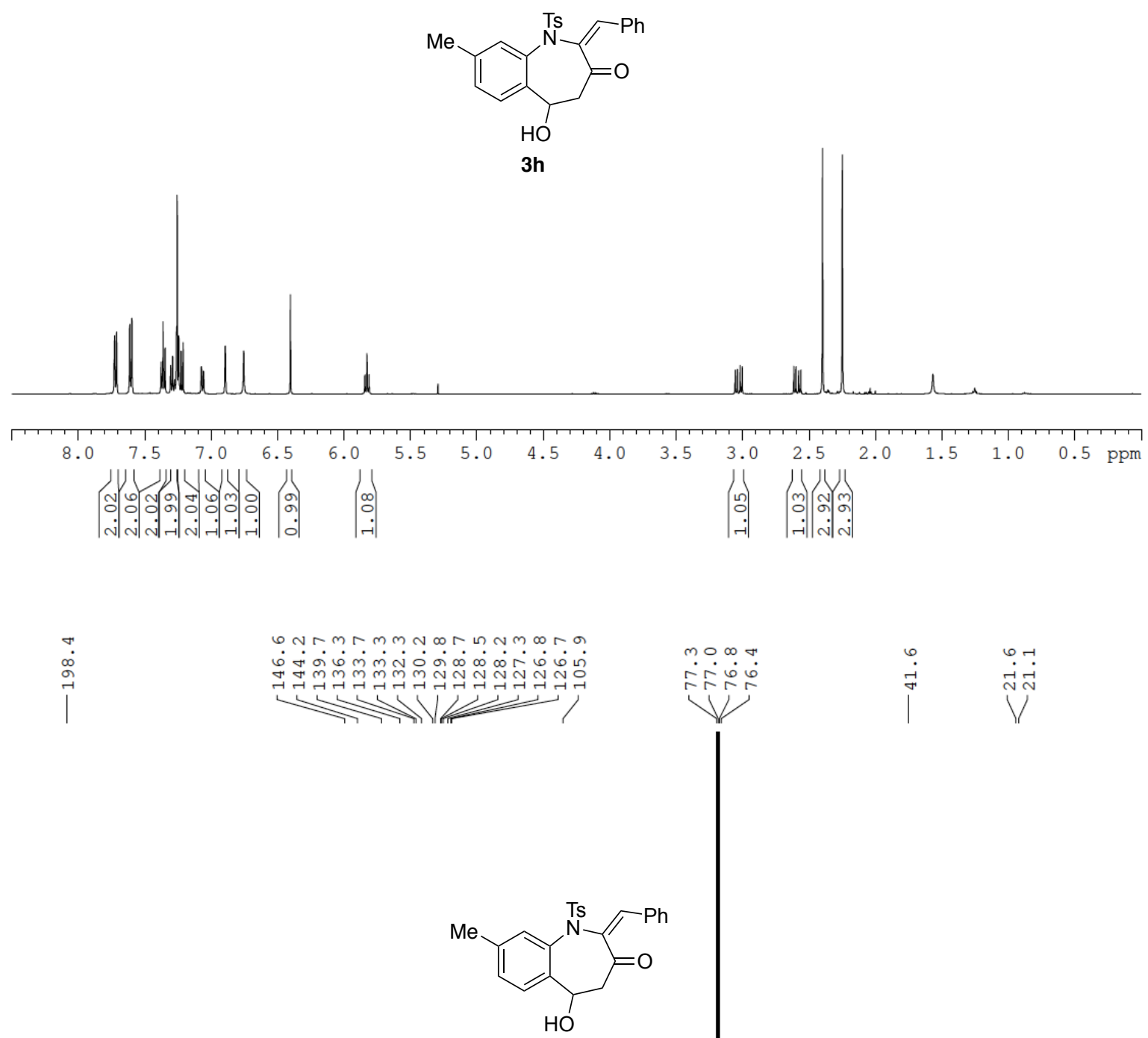

3h
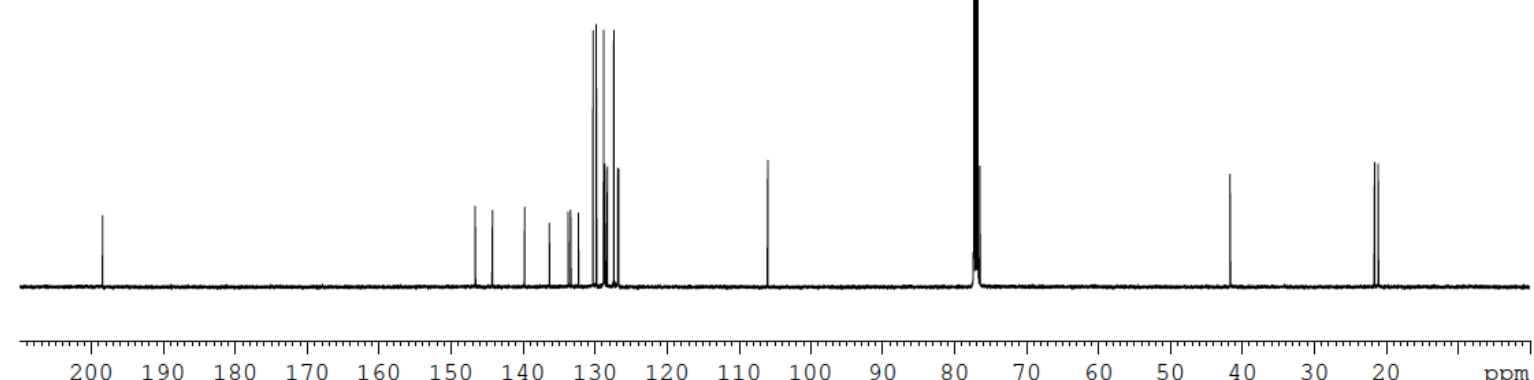

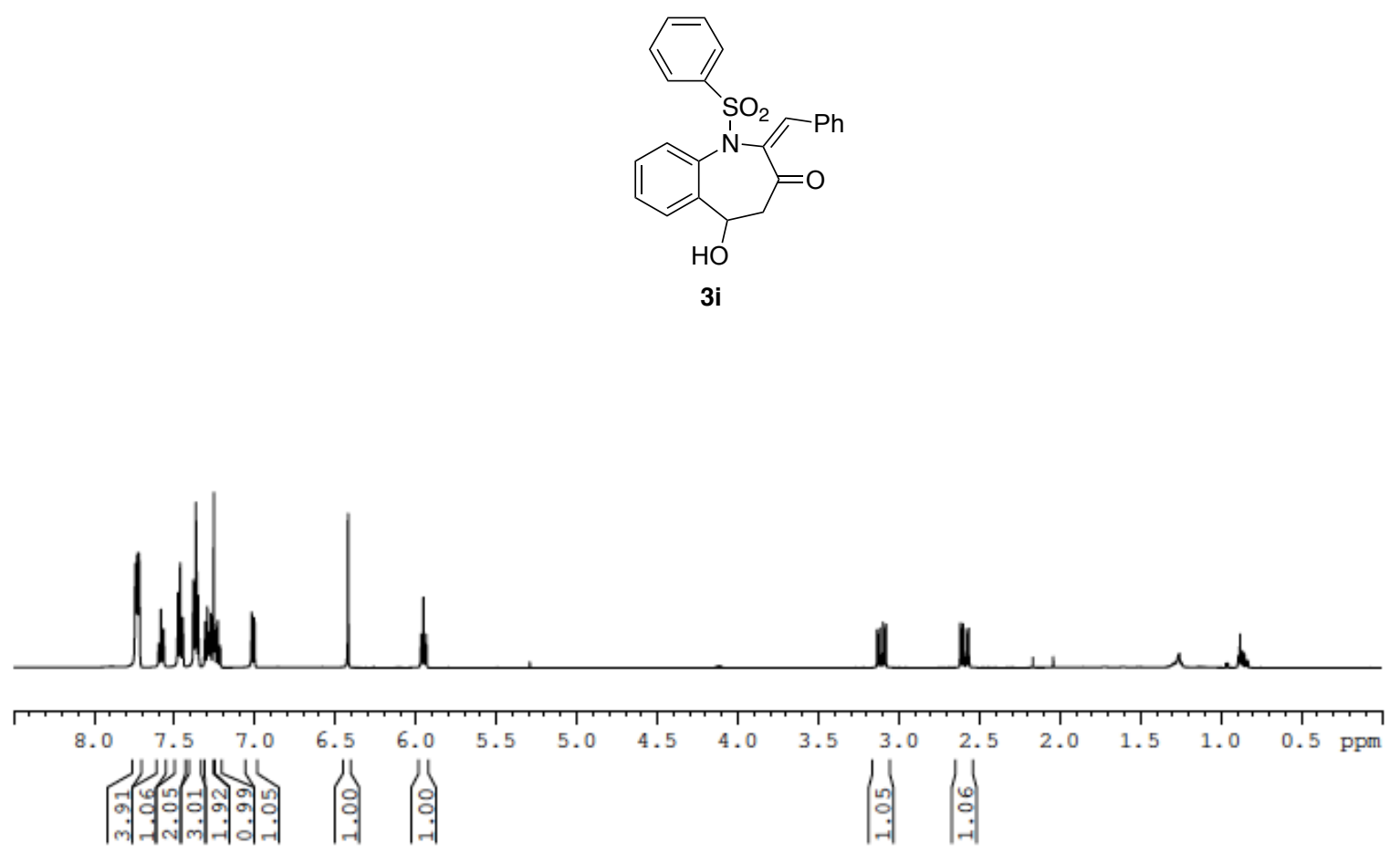

$\stackrel{m}{\infty}$
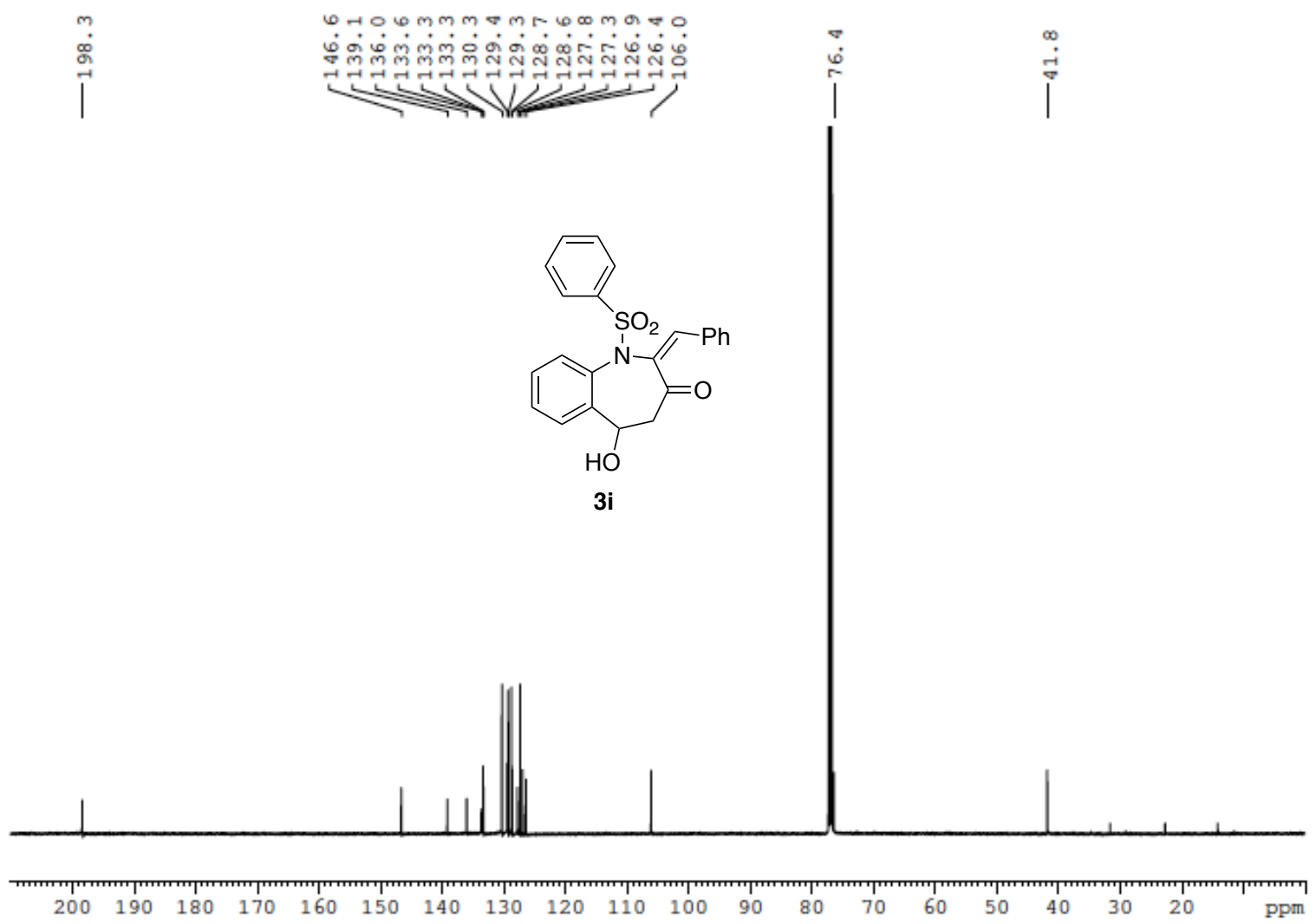


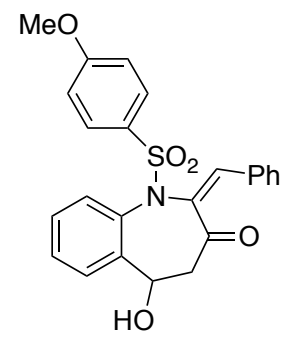

3j
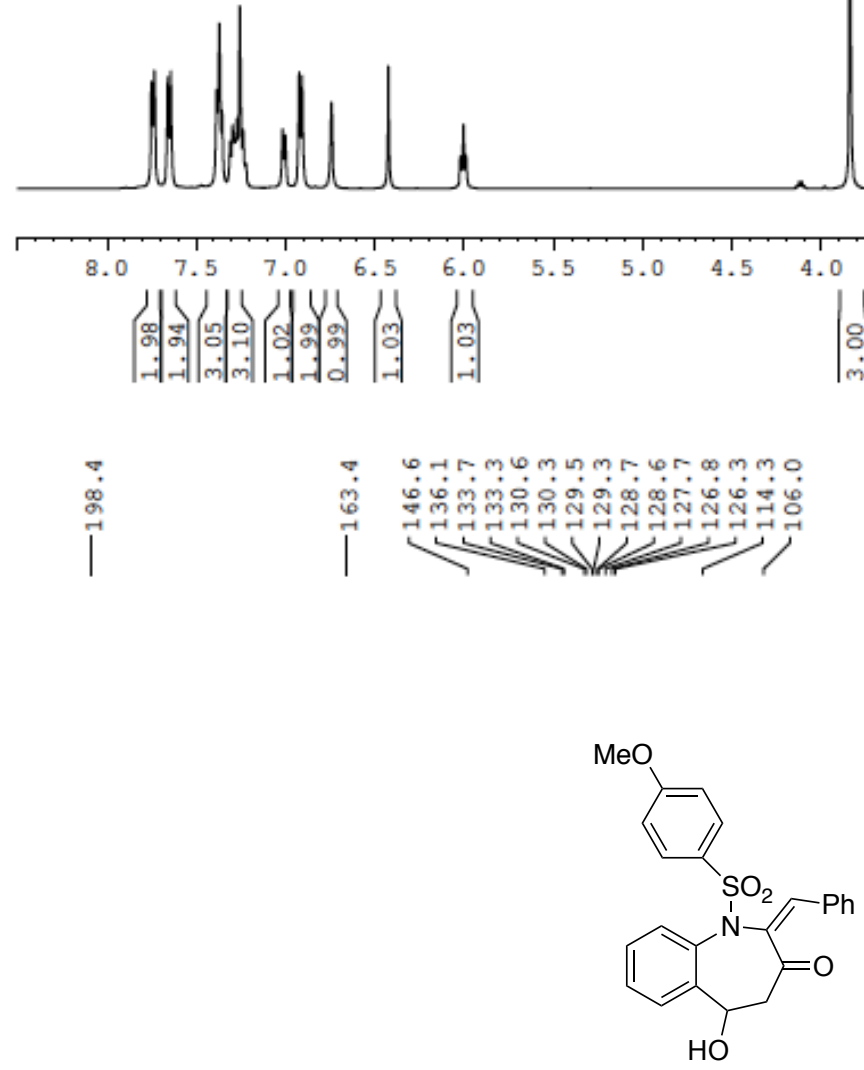

3j
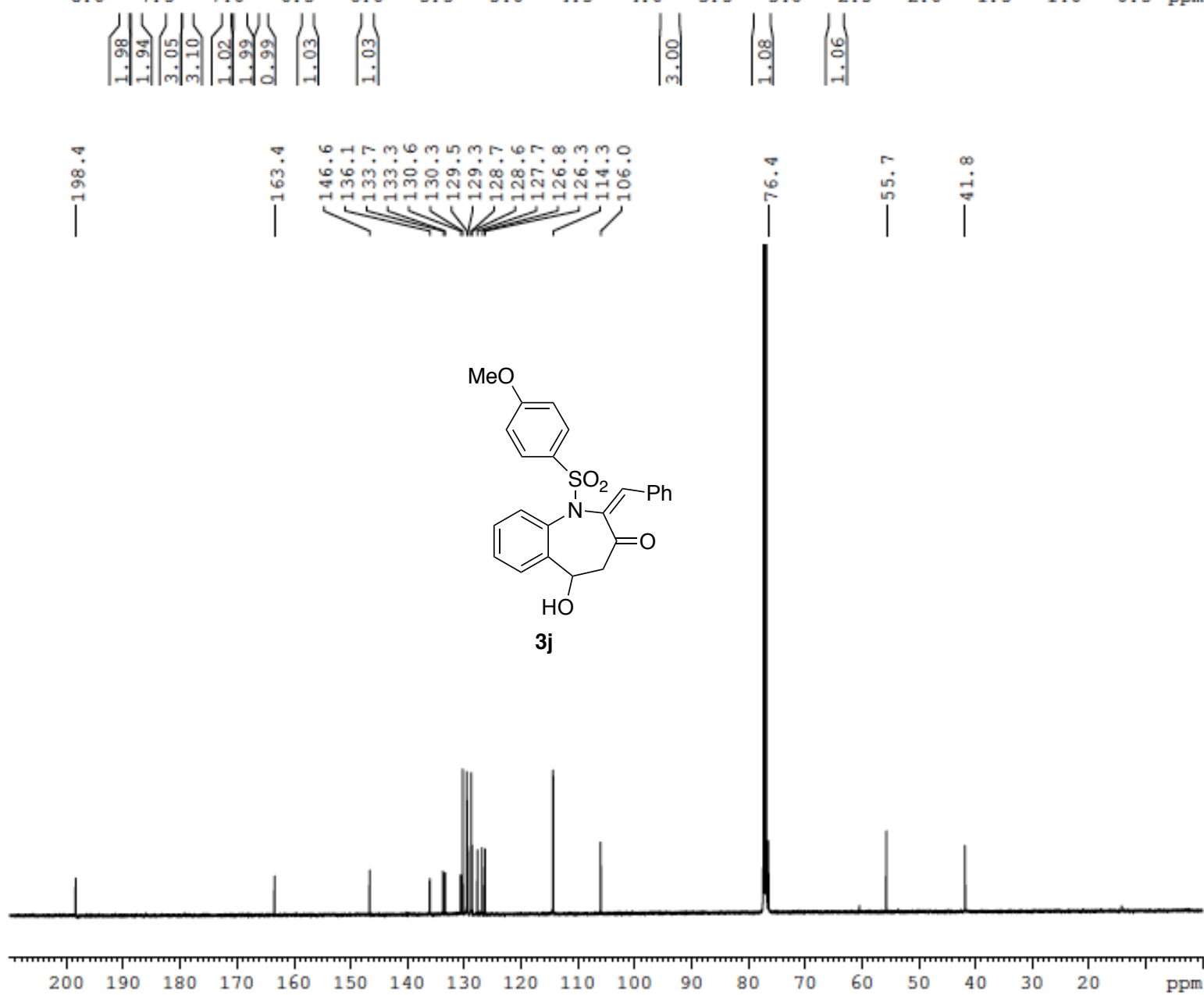

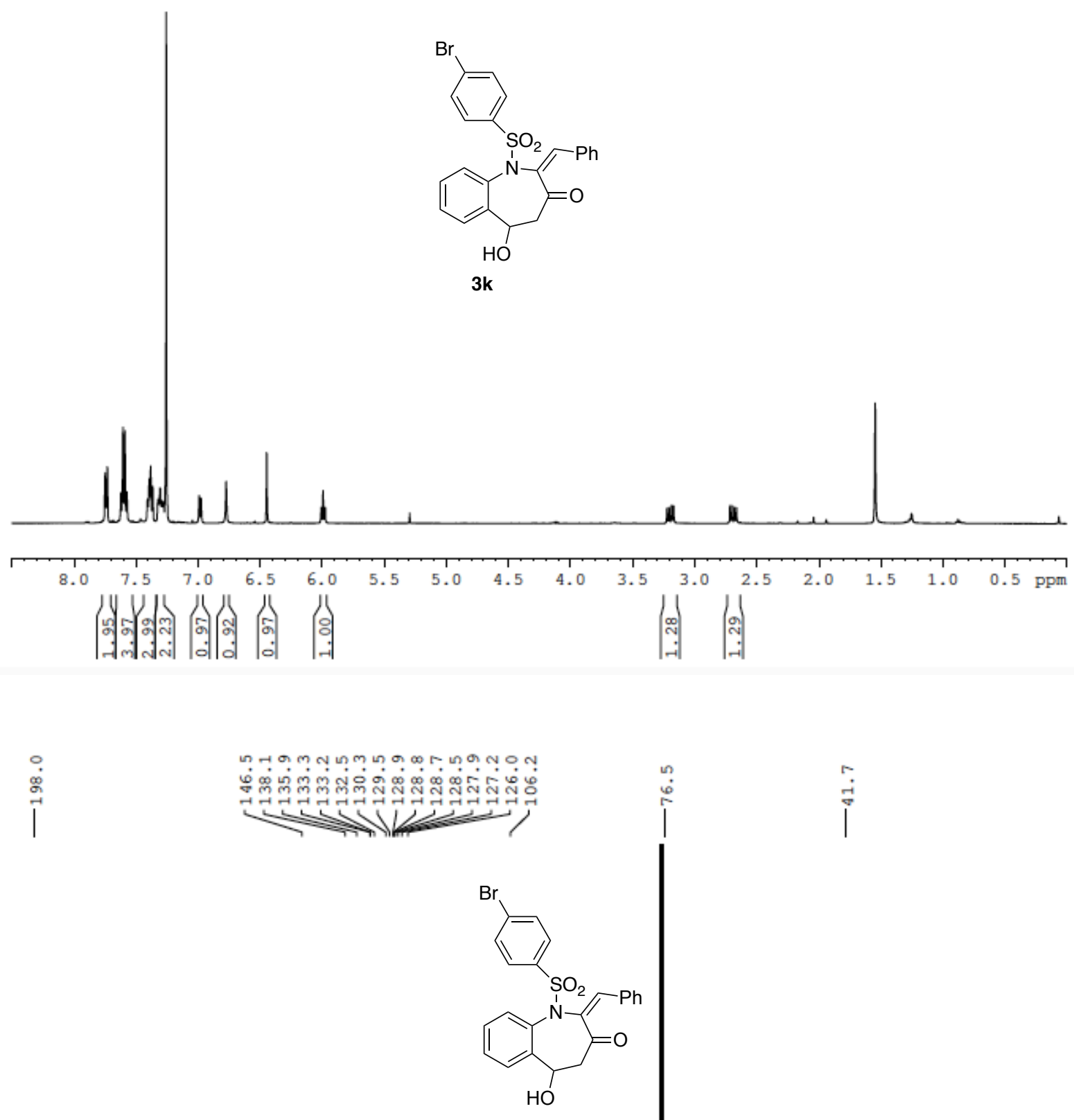

$3 k$

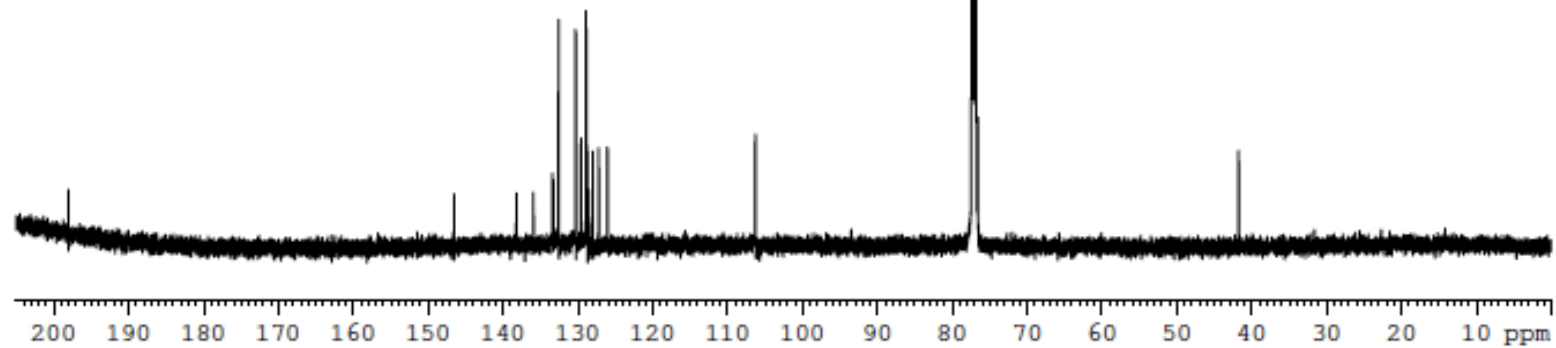



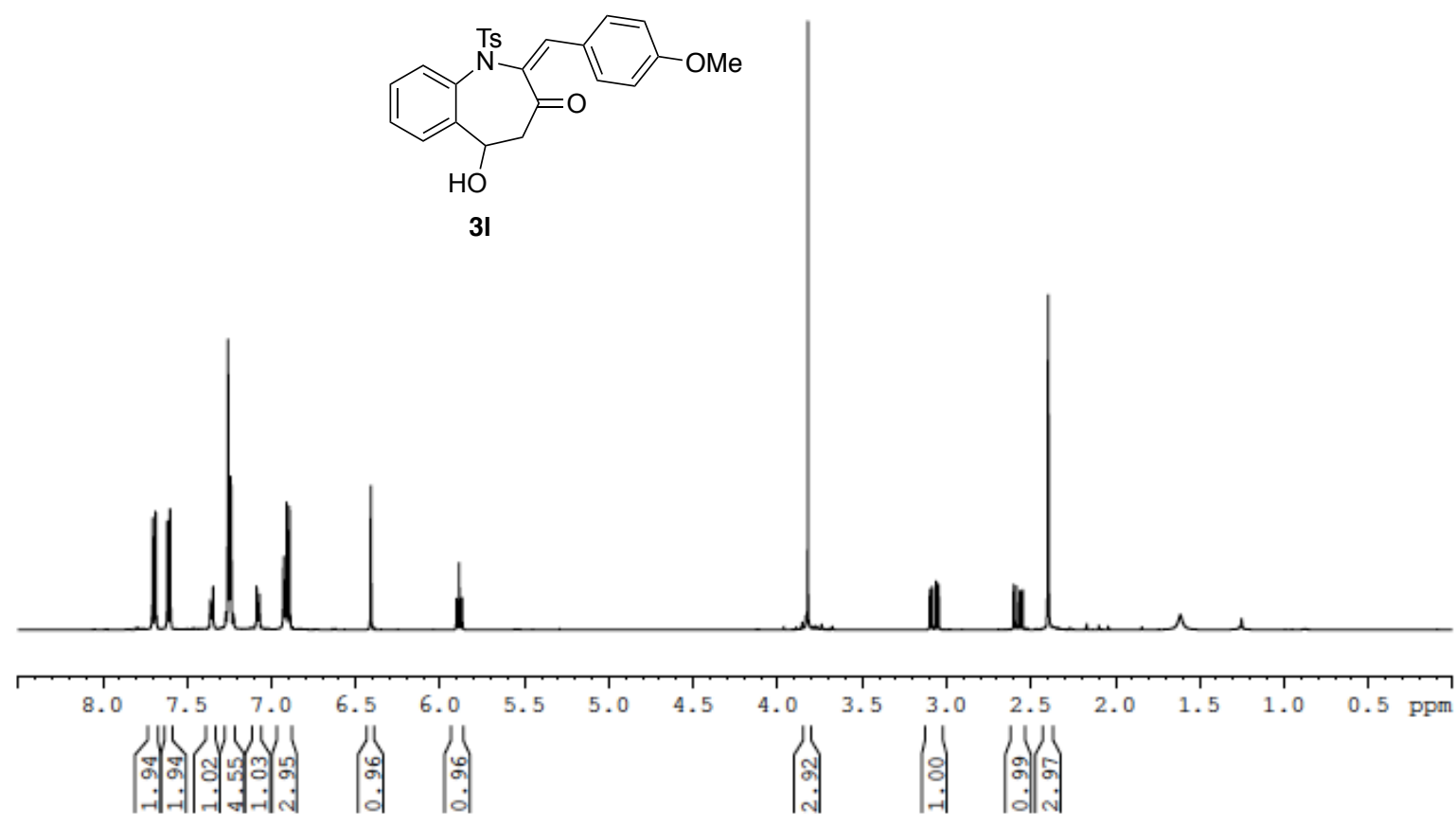

$\stackrel{0}{\stackrel{\infty}{\circ}}$
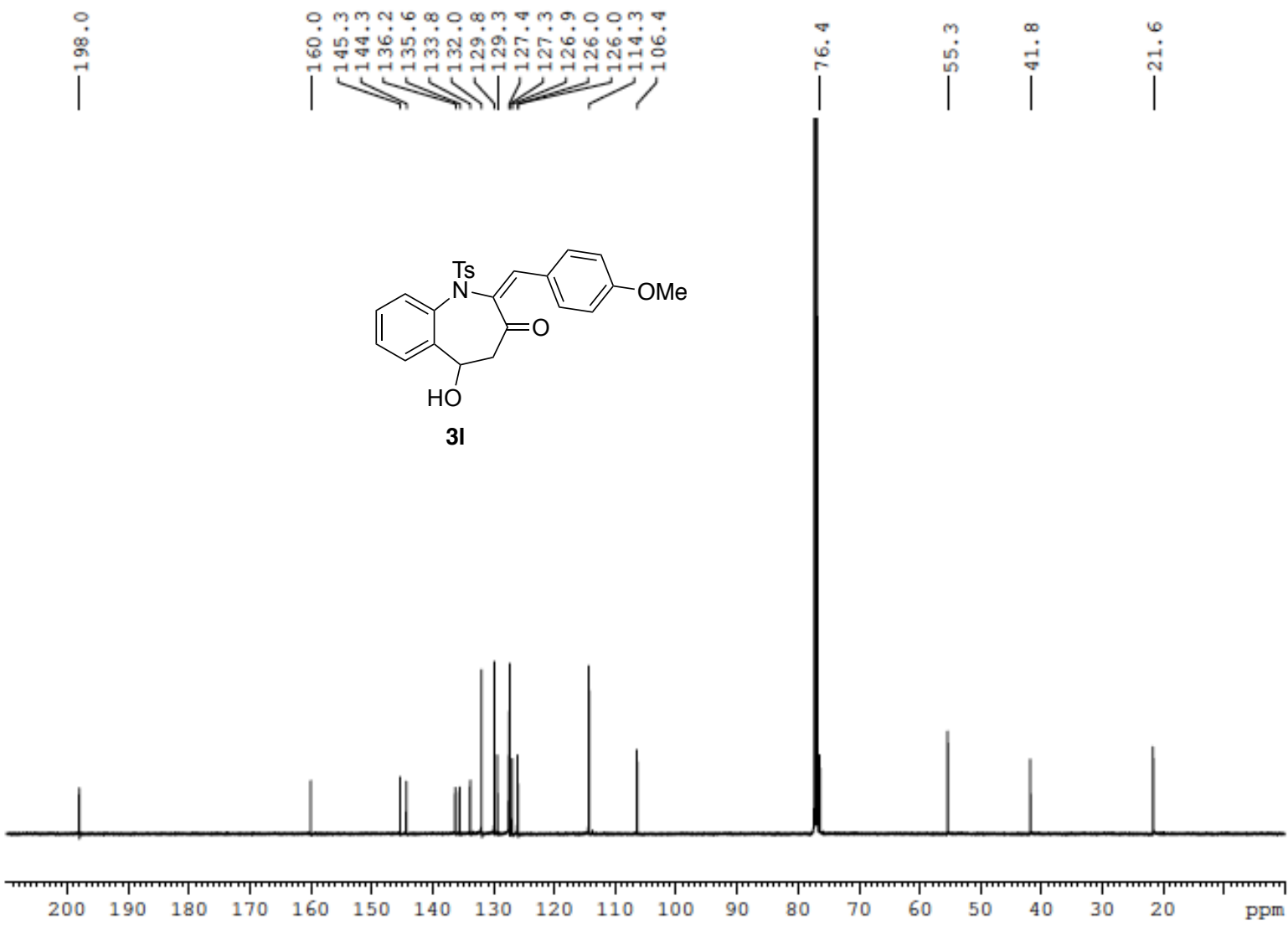


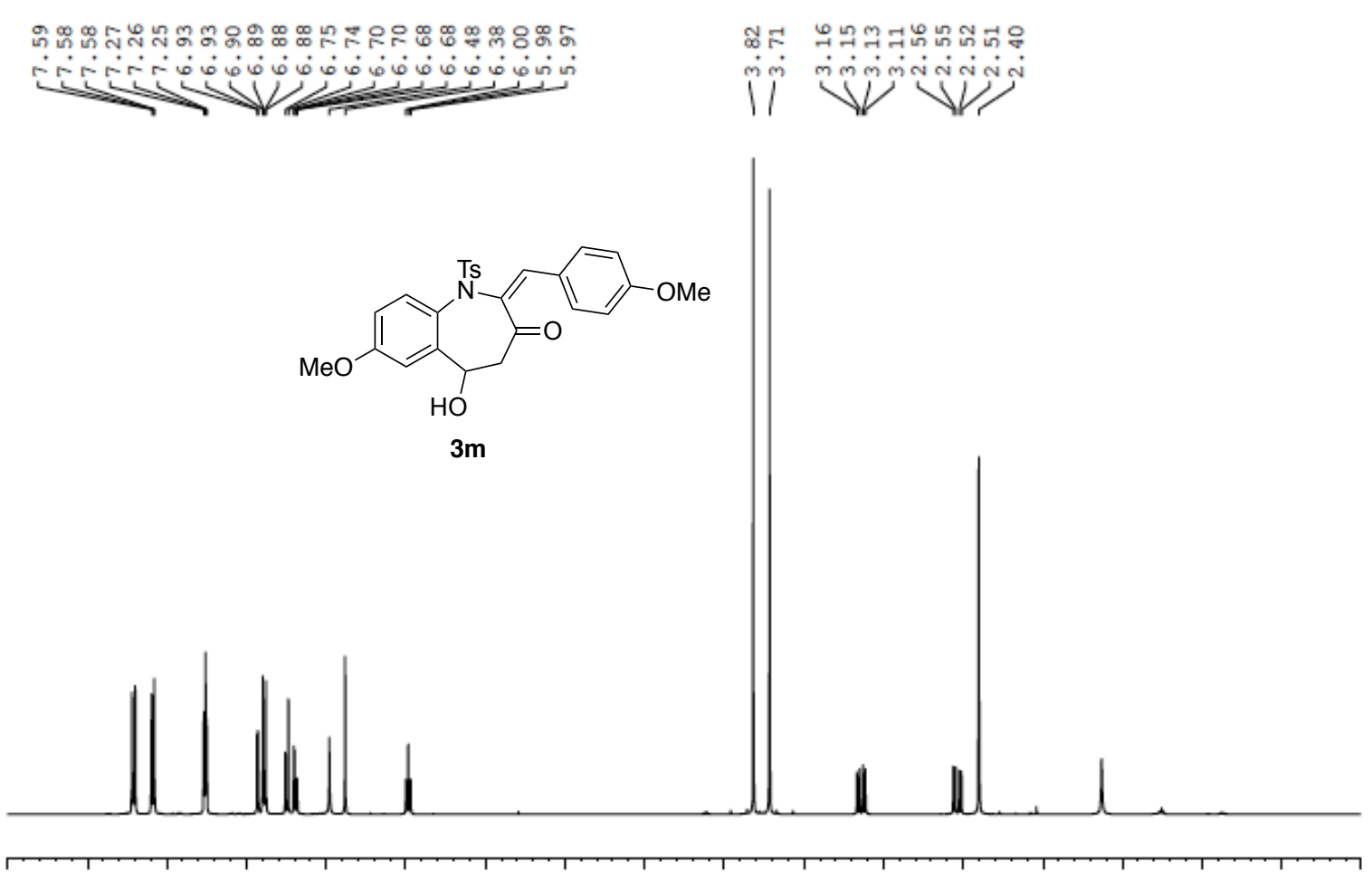

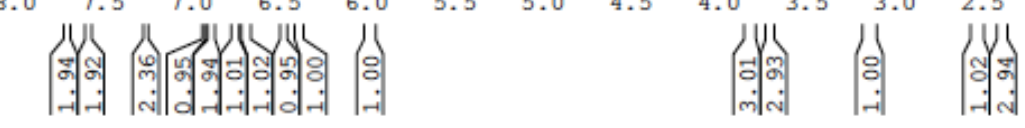
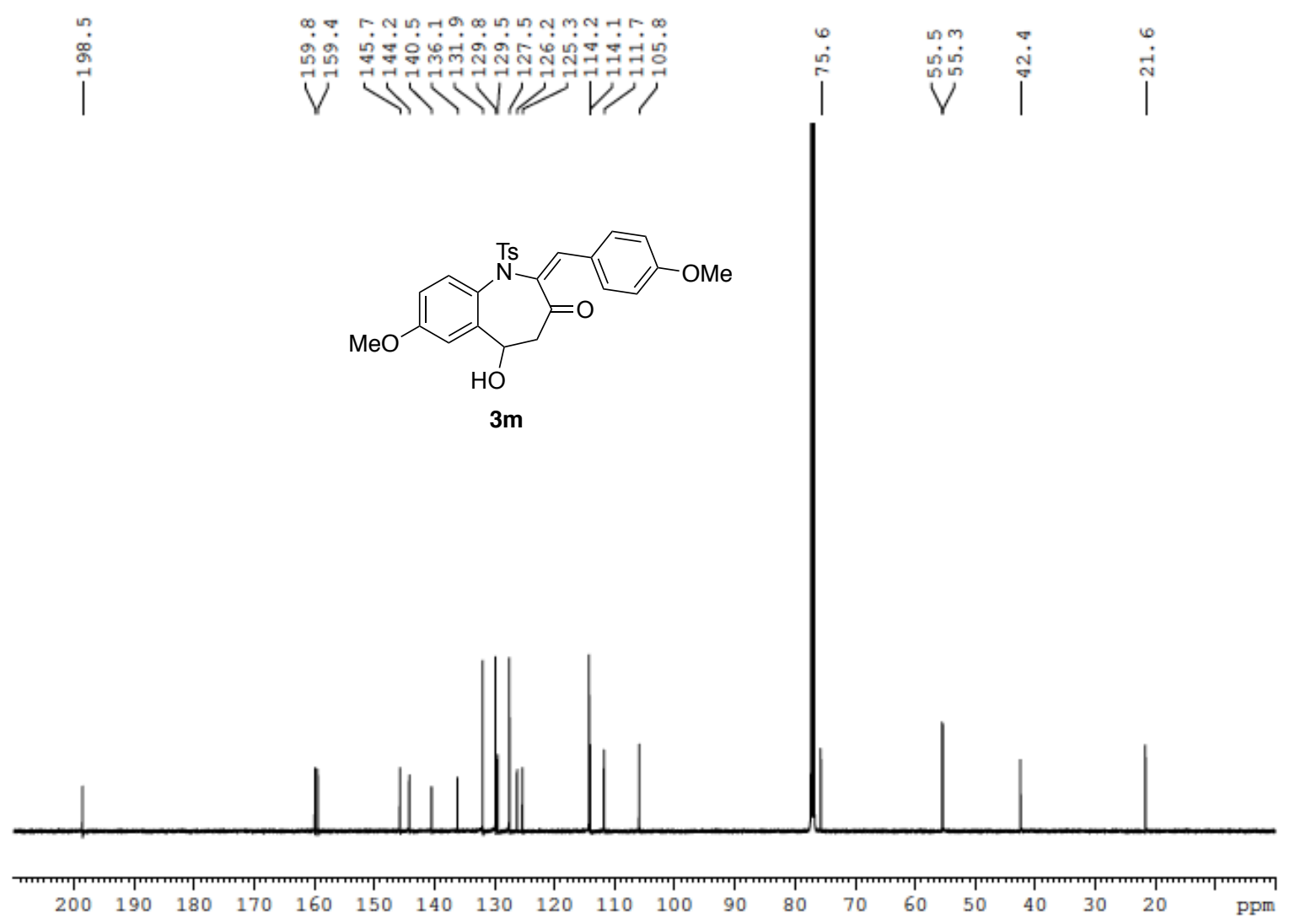


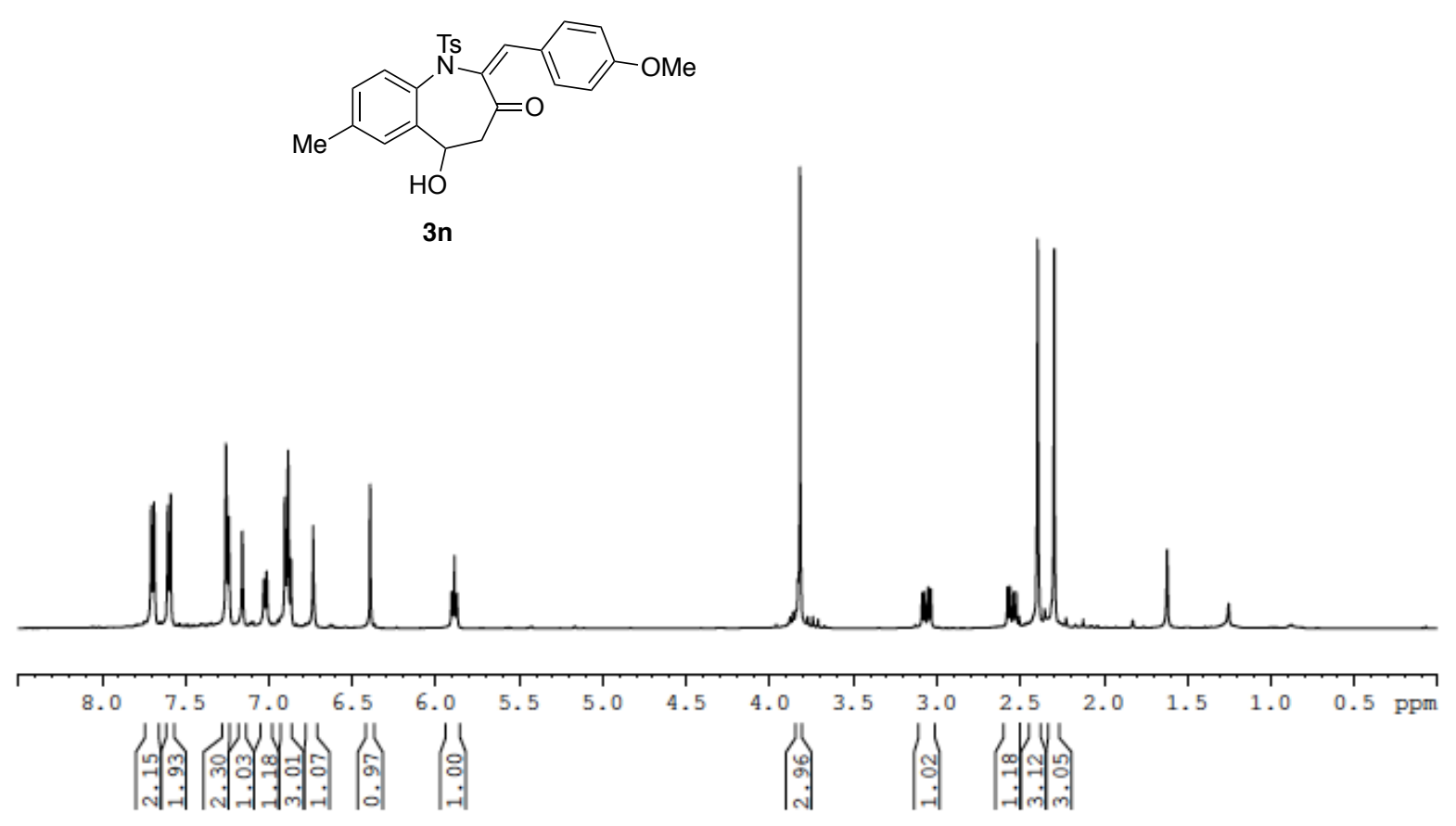

ì
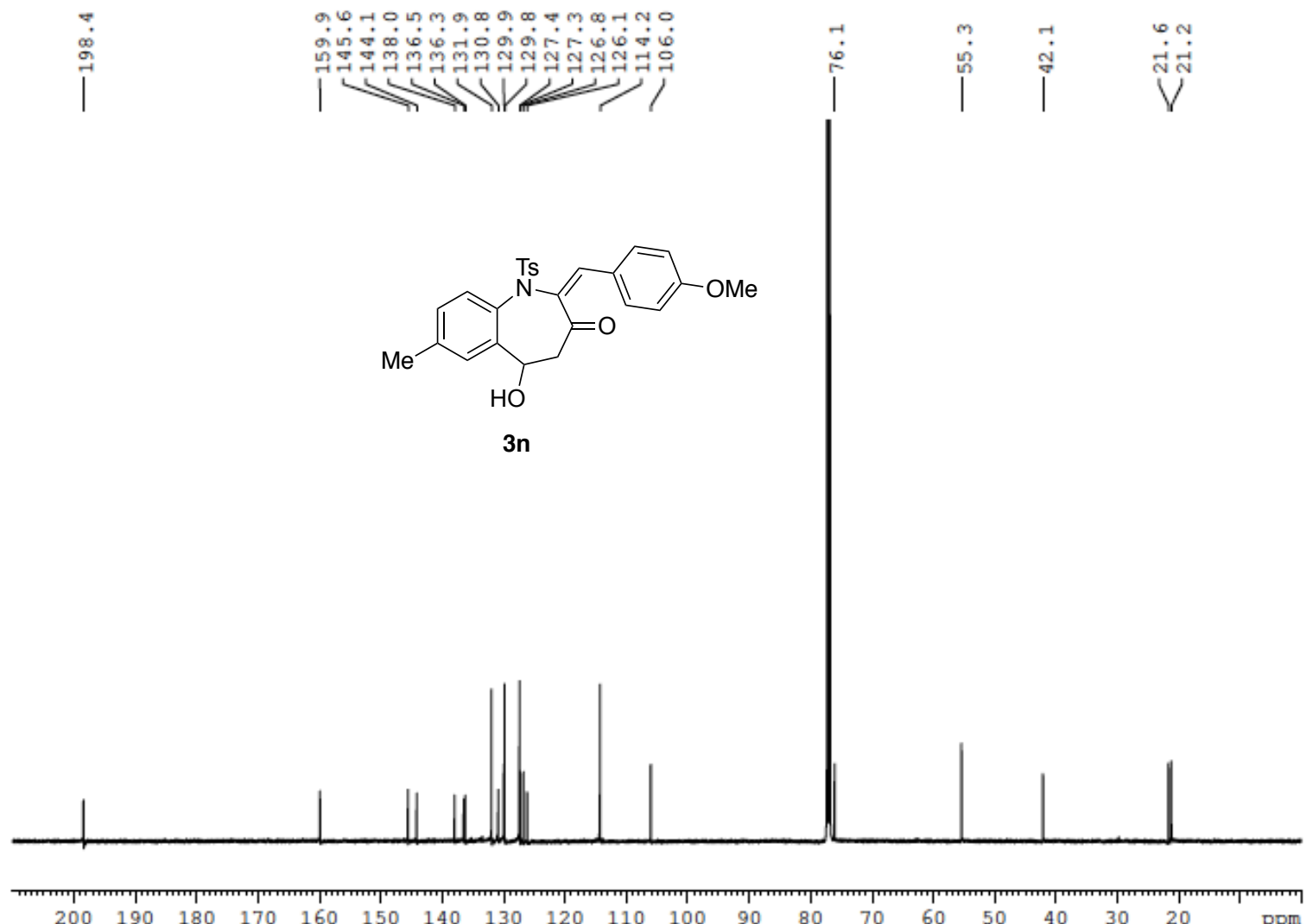

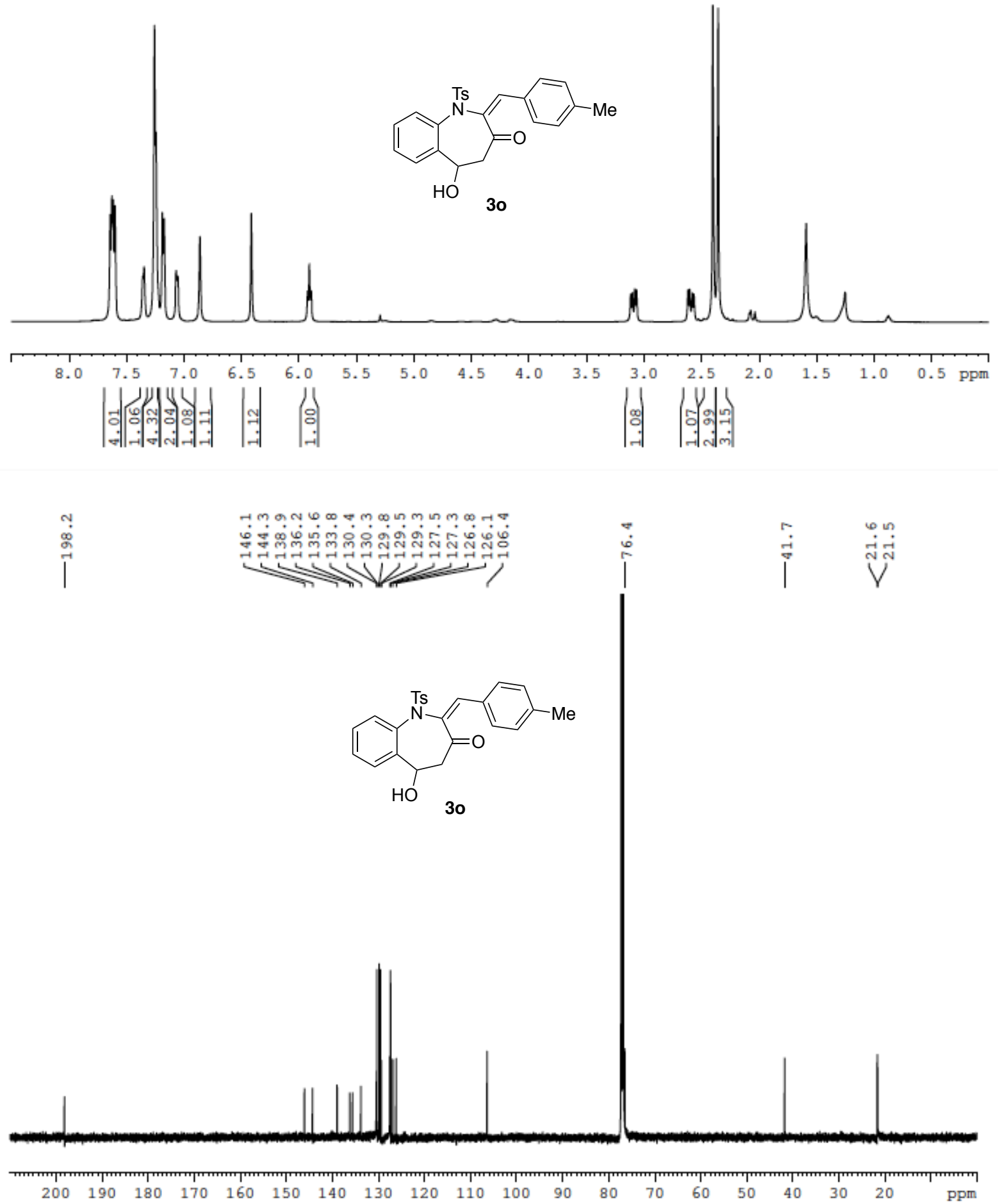

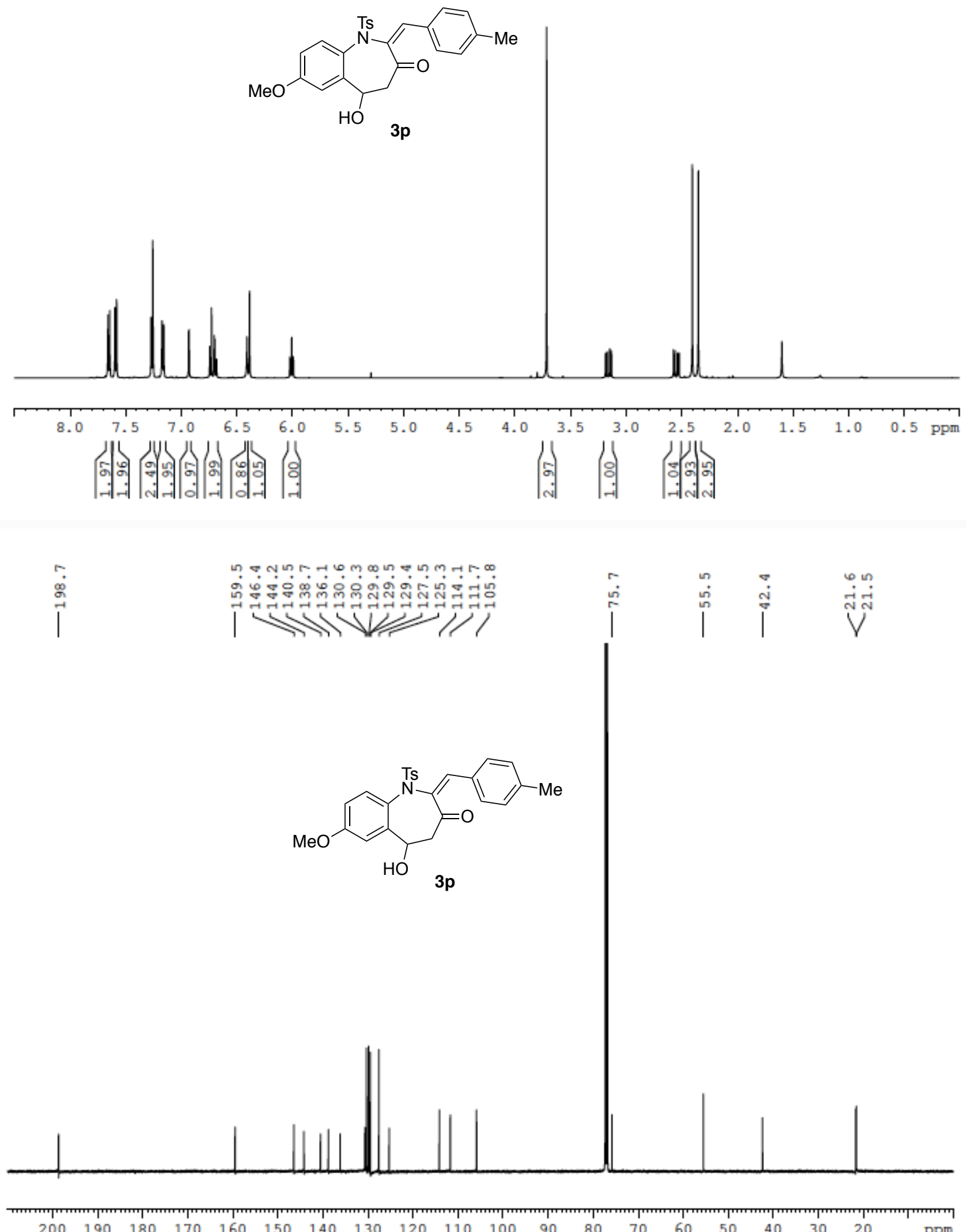

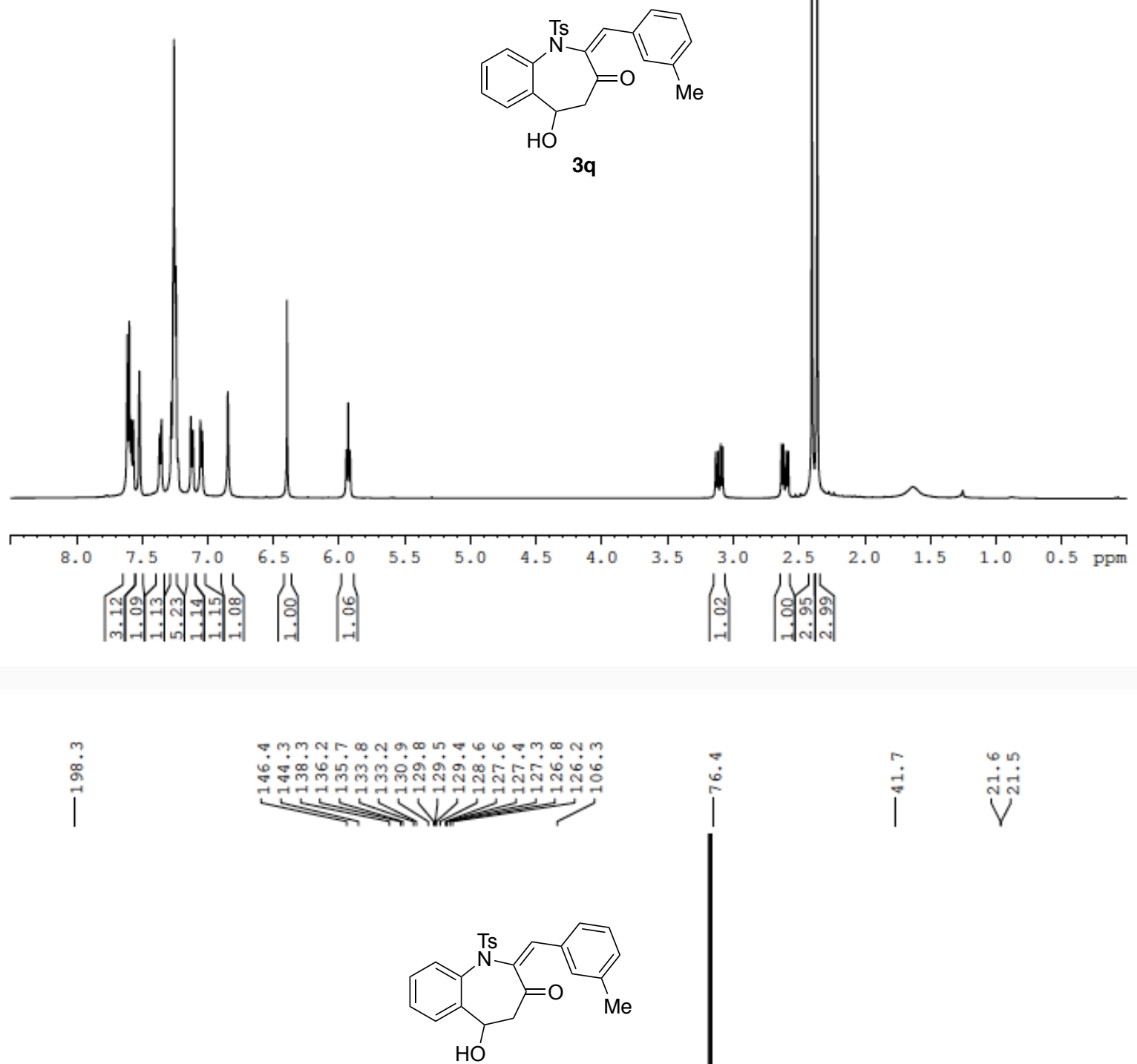

$3 q$

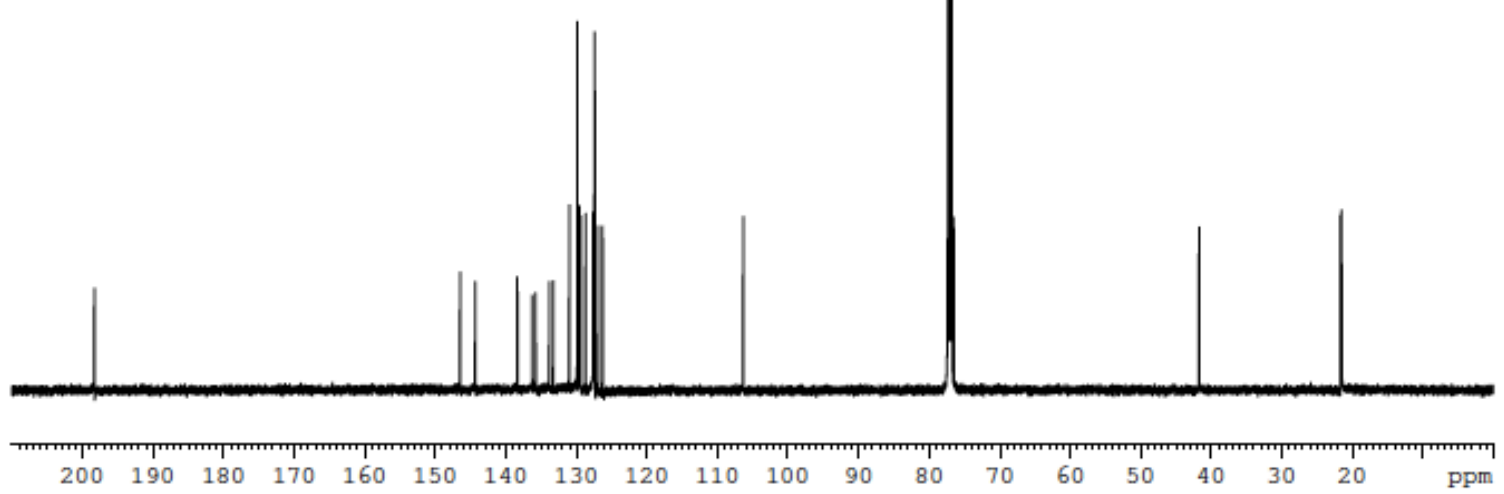


<smiles>COc1ccc2c(c1)C(O)CC(=O)C(=Cc1cccc(C)c1)[N]2</smiles>

$3 r$

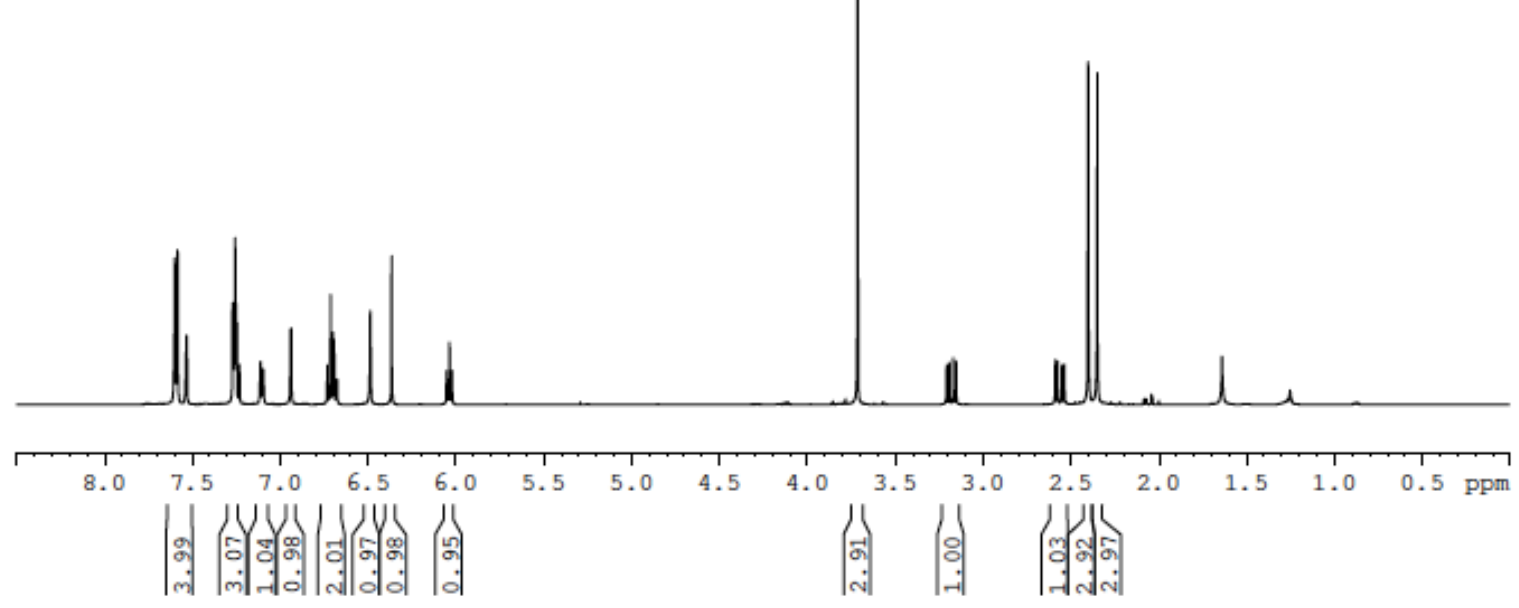

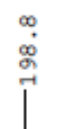

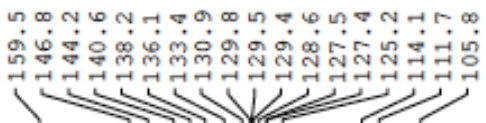

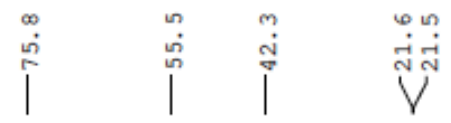<smiles>COc1ccc2c(c1)C(O)CC(=O)C2=Cc1cccc(C)c1</smiles>

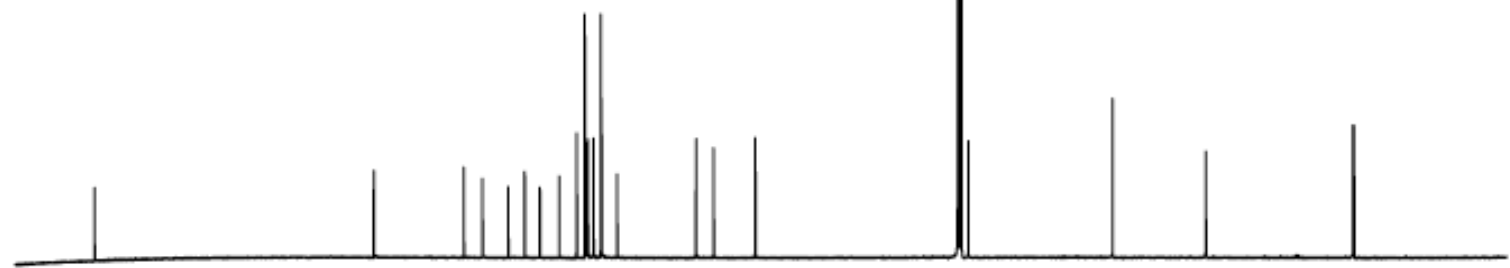

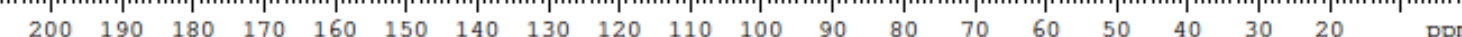



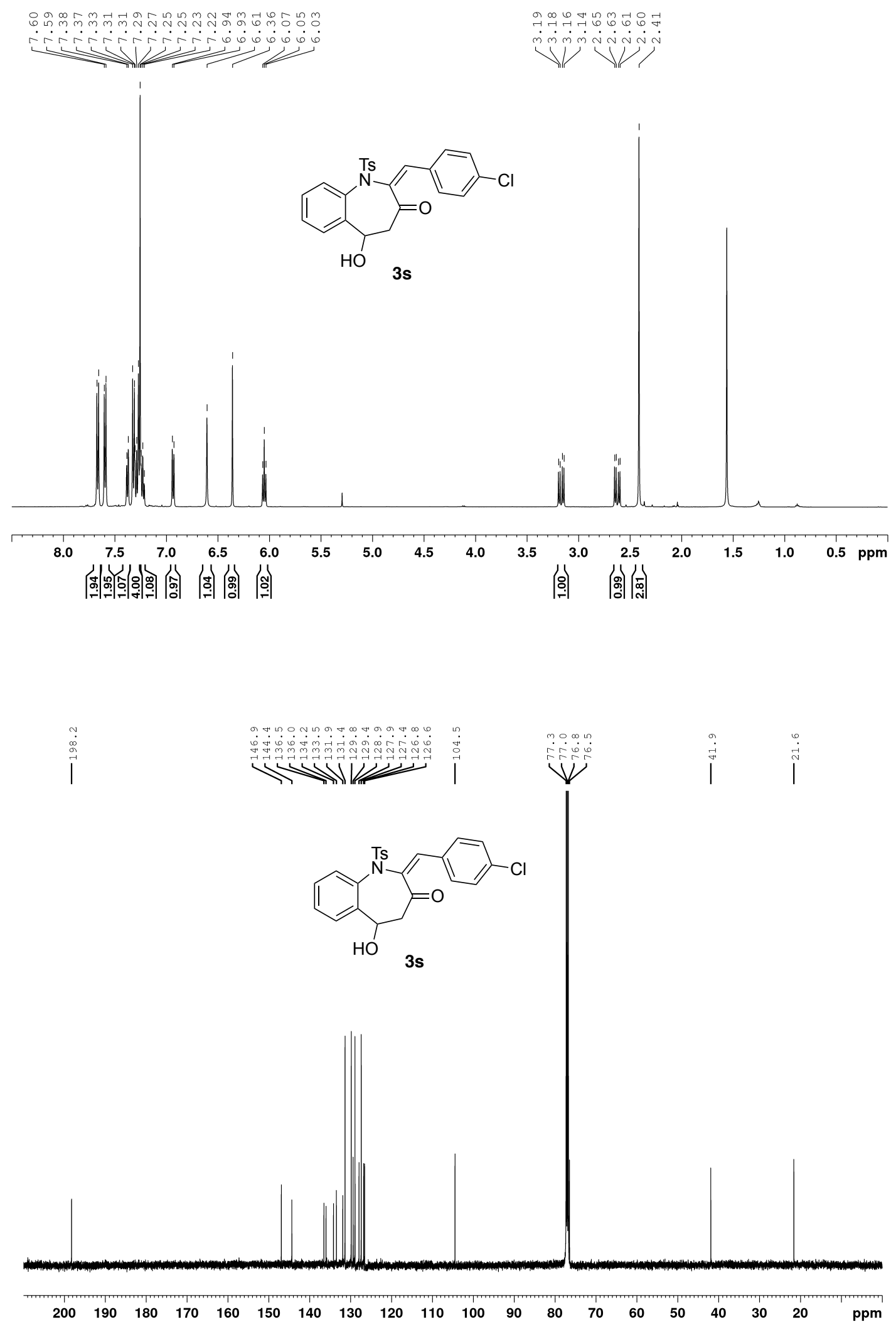

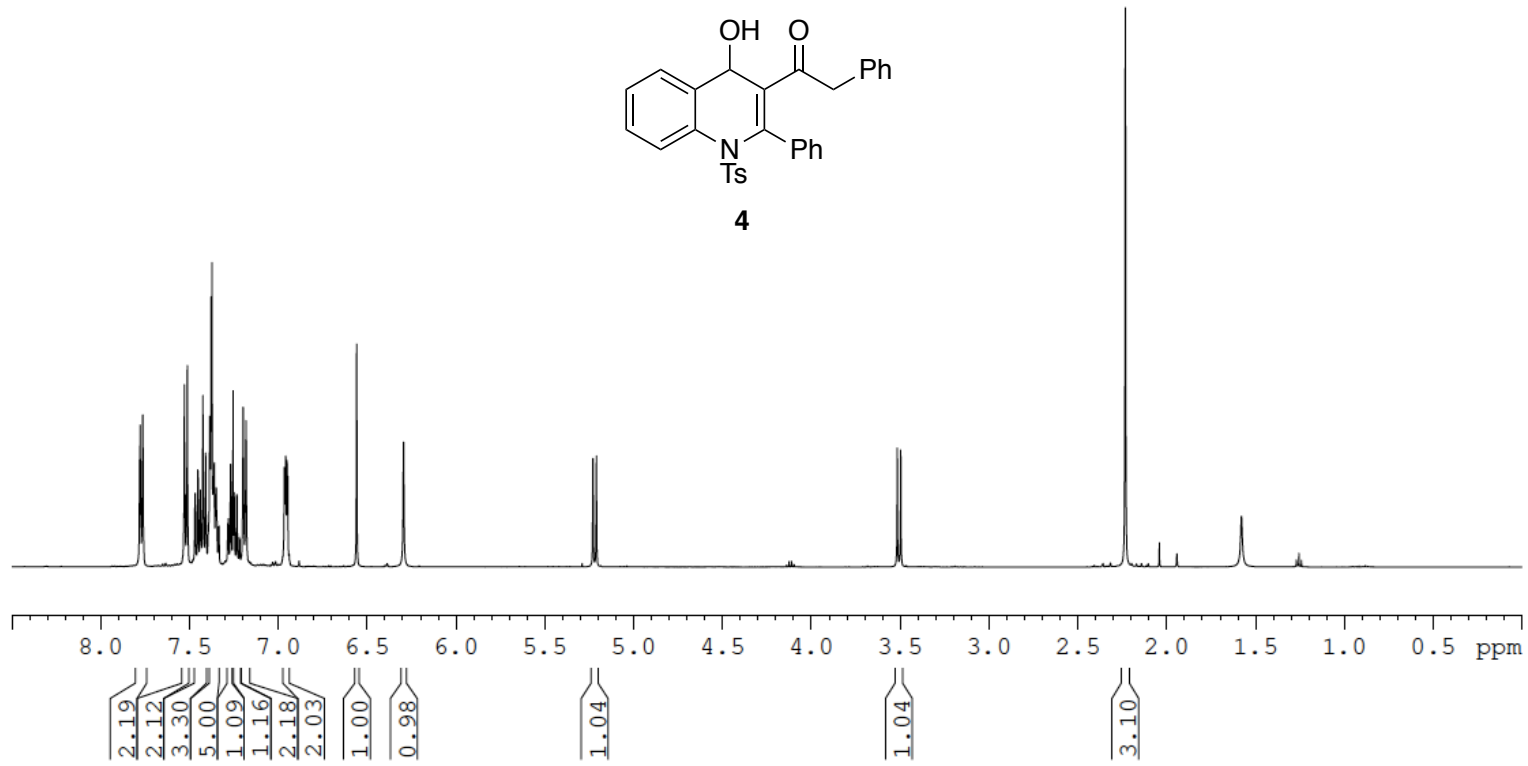

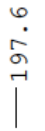
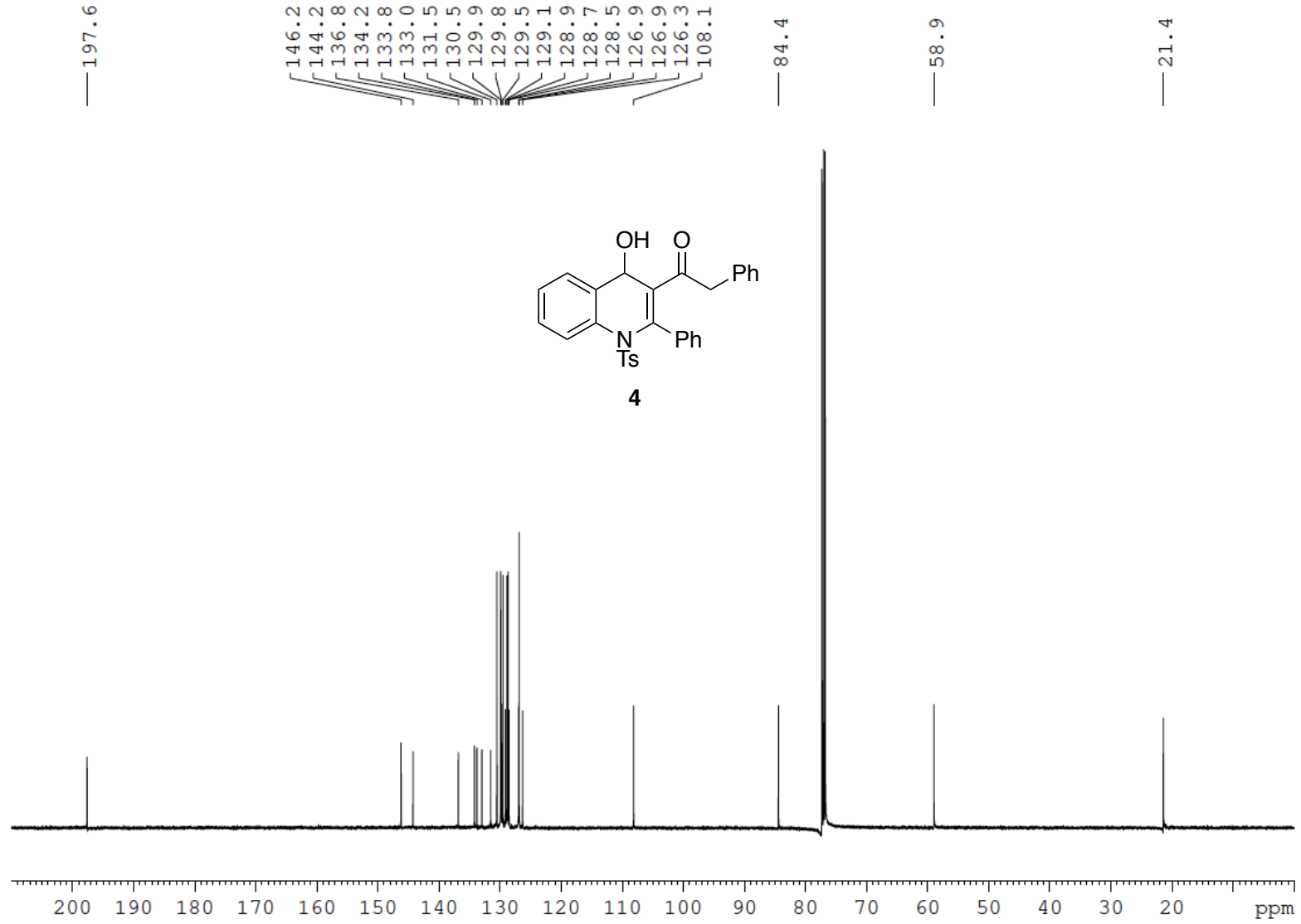

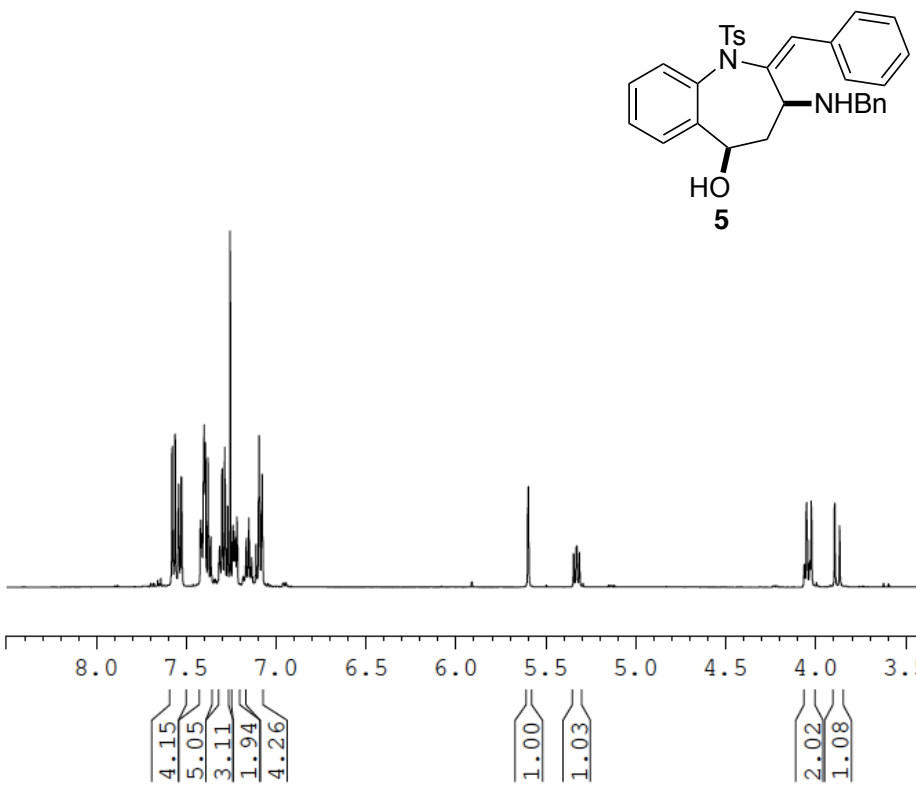

m n. . . .

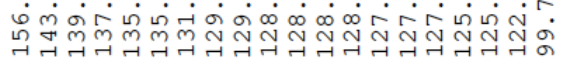
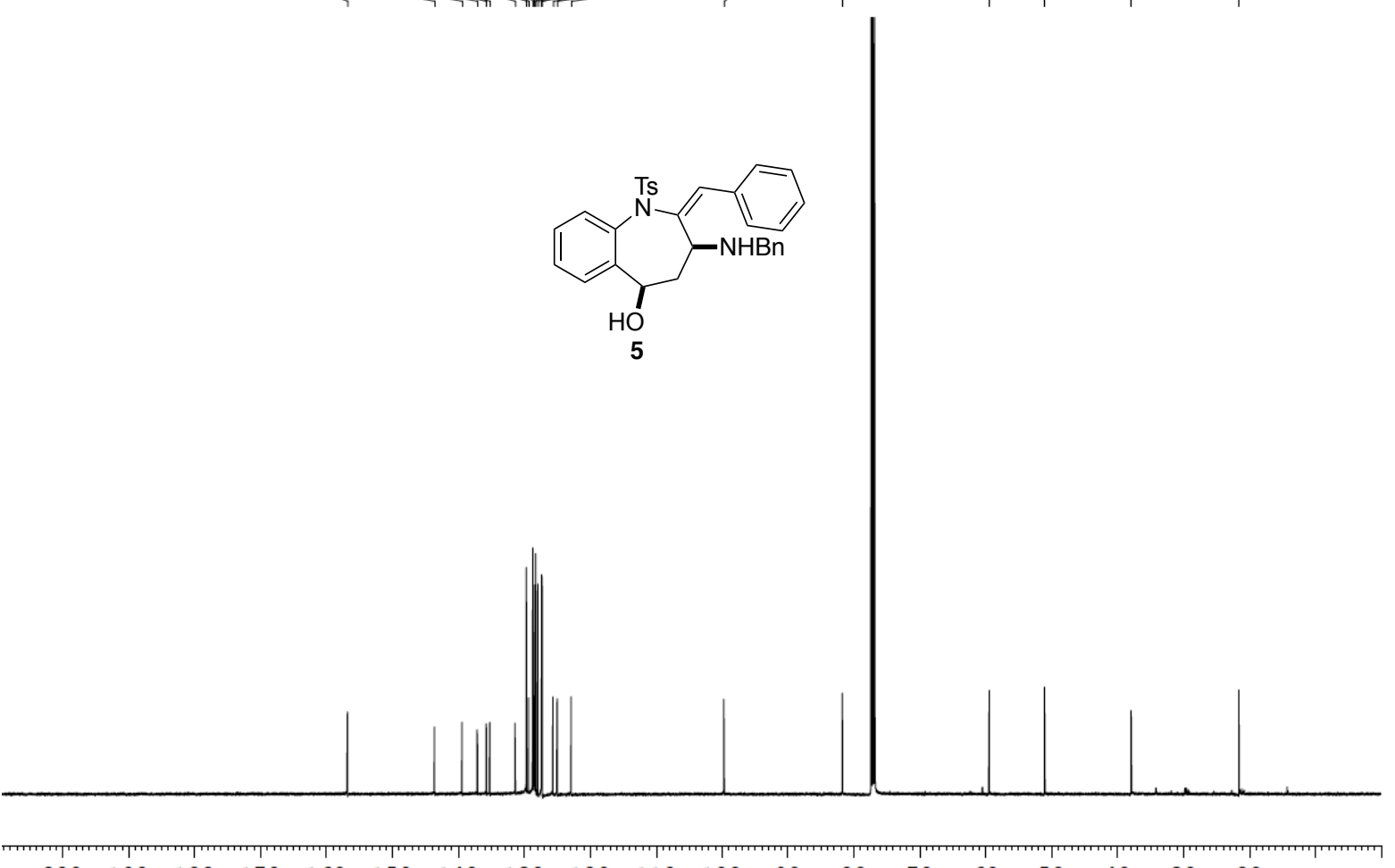

$\begin{array}{llllllllllllllllllll}200 & 190 & 180 & 170 & 160 & 150 & 140 & 130 & 120 & 110 & 100 & 90 & 80 & 70 & 60 & 50 & 40 & 30 & 20 & \mathrm{ppm}\end{array}$ 
COSY and NOESY Analysis of Compound 5.
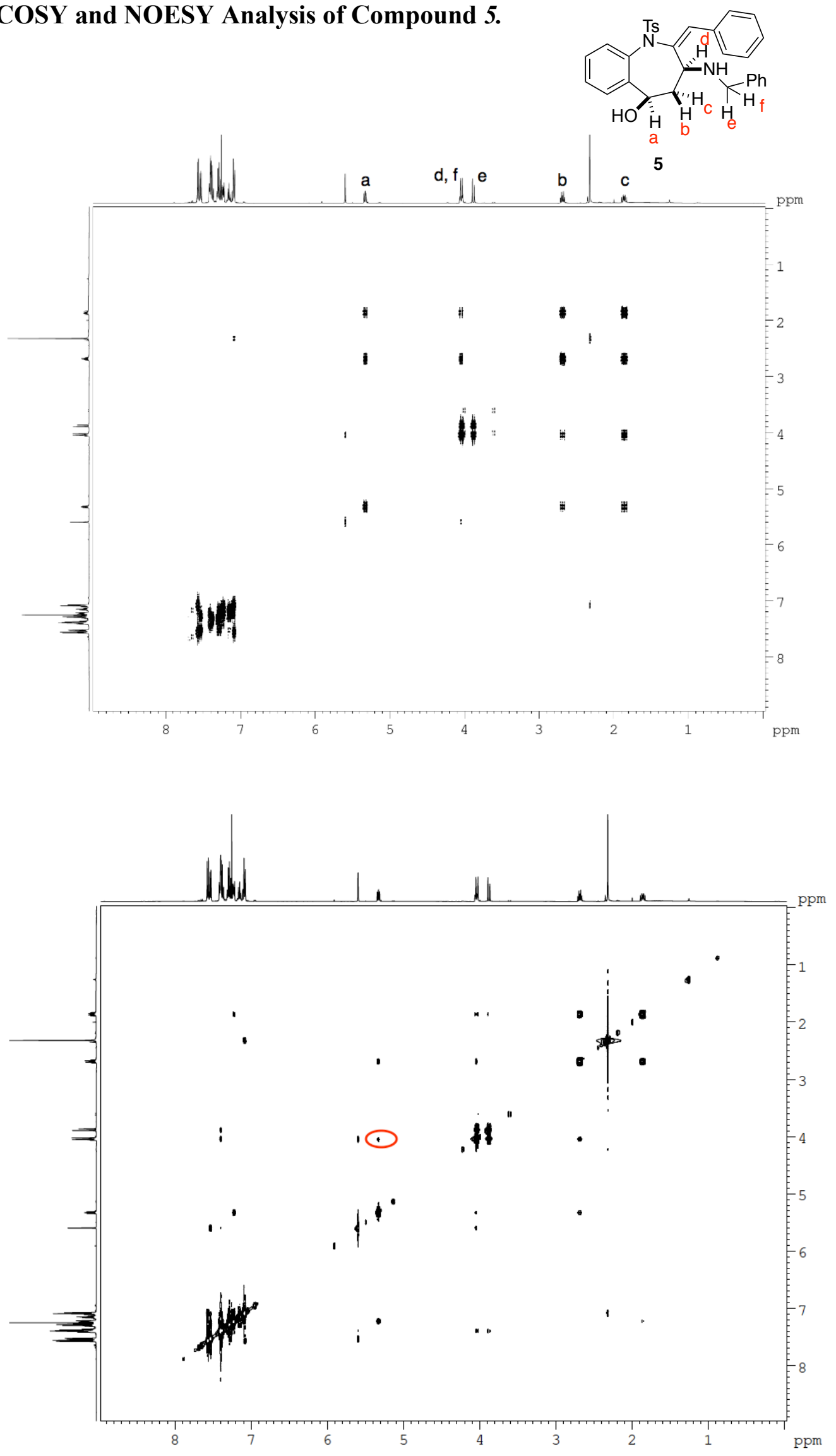

S36 

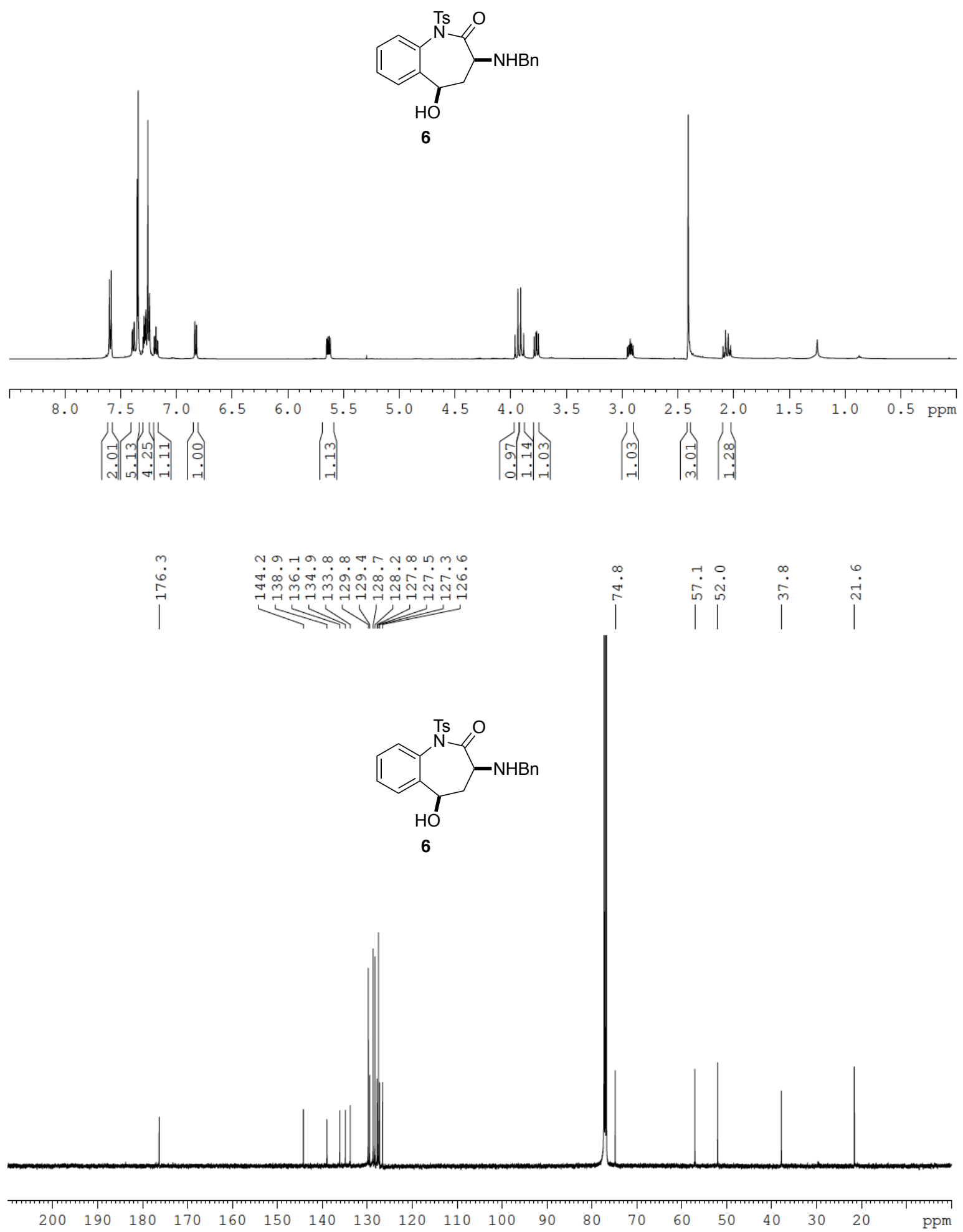